\title{
Article
}

\section{The diverse lives of massive protoplanets in self-gravitating discs}

Stamatellos, Dimitris and Inutsuka, Shu-ichiro

Available at http://clok.uclan.ac.uk/22085/

Stamatellos, Dimitris ORCID: 0000-0002-4502-8344 and Inutsuka, Shu-ichiro (2018) The diverse lives of massive protoplanets in self-gravitating discs. Monthly Notices of the Royal Astronomical Society, 477 (3). pp. 3110-3135. ISSN 0035-8711

It is advisable to refer to the publisher's version if you intend to cite from the work. http://dx.doi.org/10.1093/mnras/sty827

For more information about UCLan's research in this area go to http://www.uclan.ac.uk/researchgroups/ and search for < name of research Group>.

For information about Research generally at UCLan please go to http://www.uclan.ac.uk/research/

All outputs in CLoK are protected by Intellectual Property Rights law, including Copyright law. Copyright, IPR and Moral Rights for the works on this site are retained by the individual authors and/or other copyright owners. Terms and conditions for use of this material are defined in the policies page.

\section{CLoK}

Central Lancashire online Knowledge www.clok.uclan.ac.uk

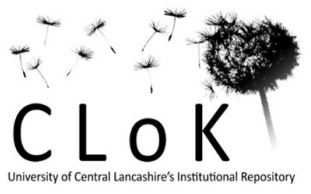




\title{
The diverse lives of massive protoplanets in self-gravitating discs
}

\author{
Dimitris Stamatellos $^{1 \star}$ and Shu-ichiro Inutsuka ${ }^{2}$ \\ ${ }_{1}^{1}$ Jeremiah Horrocks Institute for Mathematics, Physics and Astronomy, University of Central Lancashire, Preston PR1 2HE, UK \\ ${ }^{2}$ Department of Physics, Nagoya University, Chikusa-ku, Nagoya 464-8602, Japan
}

Accepted 2018 March 26. Received 2018 March 7; in original form 2017 November 21

\begin{abstract}
Gas giant planets may form early on during the evolution of protostellar discs, while these are relatively massive. We study how Jupiter-mass planet-seeds (termed protoplanets) evolve in massive, but gravitationally stable $(Q \gtrsim 1.5)$, discs using radiative hydrodynamic simulations. We find that the protoplanet initially migrates inwards rapidly, until it opens up a gap in the disc. Thereafter, it either continues to migrate inwards on a much longer time-scale or starts migrating outwards. Outward migration occurs when the protoplanet resides within a gap with gravitationally unstable edges, as a high fraction of the accreted gas is high angular momentum gas from outside the protoplanet's orbit. The effect of radiative heating from the protoplanet is critical in determining the direction of the migration and the eccentricity of the protoplanet. Gap opening is facilitated by efficient cooling that may not be captured by the commonly used $\beta$-cooling approximation. The protoplanet initially accretes at a high rate $\left(\sim 10^{-3} M_{\mathrm{J}} \mathrm{yr}^{-1}\right)$, and its accretion luminosity could be a few tenths of the host star's luminosity, making the protoplanet easily observable (albeit only for a short time). Due to the high gas accretion rate, the protoplanet generally grows above the deuterium-burning mass-limit. Protoplanet radiative feedback reduces its mass growth so that its final mass is near the brown dwarfplanet boundary. The fate of a young planet-seed is diverse and could vary from a gas giant planet on a circular orbit at a few au from the central star to a brown dwarf on an eccentric, wide orbit.
\end{abstract}

Key words: accretion, accretion discs-hydrodynamics-protoplanetary discs-brown dwarfs - planetary systems.

\section{INTRODUCTION}

Observational advances over the last $20 \mathrm{yr}$ have made possible the discovery of a large number of exoplanets (see the 'Extrasolar Planets Encyclopaedia'; http://exoplanet.eu). Most of these exoplanets are gas giants, as these are easier to detect with the main current methods (radial velocity, transits) than small, rocky planets like Earth.

Two theoretical models have been proposed for the formation of gas giant planets: (i) core accretion, and (ii) gravitational fragmentation of protostellar discs.

In the core accretion model (Safronov 1972; Goldreich \& Ward 1973; Mizuno 1980; Bodenheimer \& Pollack 1986; Pollack et al. 1996), the cores of giant planets form in circumstellar discs by coagulation of dust particles to progressively larger objects, until a core with a few Earth masses has formed. Such a core subsequently accretes an envelope of gas from the disc. One of the main drawbacks of this model is that, in its standard formulation, it requires a few million years to form gas giants, a time-scale which may

^E-mail: dstamatellos@uclan.ac.uk be long when compared to the observed lifetimes of circumstellar discs (of the order of a few Myr; Haisch, Lada \& Lada 2001; Hernández et al. 2008; Muzerolle et al. 2010). Moreover, this model seems unlikely to be able to produce massive planets on wide orbits (Kraus et al. 2008, 2014; Marois et al. 2008; Faherty et al. 2009; Ireland et al. 2011; Kuzuhara et al. 2011, 2013; Aller et al. 2013; Rameau et al. 2013; Bailey et al. 2014; Galicher et al. 2014; Naud et al. 2014), like e.g. the planets around HR8799.

A second way to form gas giants is by gravitational fragmentation of protostellar discs (Kuiper 1951; Cameron 1978; Boss 1997). This model circumvents the time-scale problem of the core accretion theory, as planets form on a dynamical time-scale, i.e. within a few $10^{3} \mathrm{yr}$. One of the main issues relating to this model is whether protostellar discs are able to fragment or not. There are two criteria for disc fragmentation: (i) the Toomre criterion (Toomre 1964) that postulates that the disc must be massive enough in order gravity to dominate over the local thermal and centrifugal support, and (ii) the Gammie criterion (Gammie 2001; Johnson \& Gammie 2003; Rice, Lodato \& Armitage 2005) that asserts that the disc must be able to cool fast enough, i.e. on a dynamical time-scale (see Takahashi, Tsukamoto \& Inutsuka 2016a, for an alternative view). There has been relative consensus that these two criteria can be 
satisfied at distances $>50-100$ au from the star hosting the disc; therefore, discs can fragment at such distances (Matzner \& Levin 2005; Rafikov 2005; Boley et al. 2006; Whitworth \& Stamatellos 2006; Stamatellos \& Whitworth 2008). This fact, together with the discovery of exoplanets on wide orbits, may suggest two modes of gas giant planet formation: core accretion that forms planets close to the host star, and disc fragmentation that forms planets on wide orbits (Boley 2009).

Many studies suggesting that planets form by disc fragmentation, refer to the initial mass of the fragments produced. The initial fragment mass (e.g. Stamatellos \& Whitworth 2009a,b) is determined by the opacity limit for fragmentation, i.e. the minimum mass a fragment produced by fragmenting gas may have (e.g. Low \& Lynden-Bell 1976; Rees 1976; Silk 1977; Boss 1988; Masunaga \& Inutsuka 1999; Whitworth \& Stamatellos 2006). However, once a fragment forms in a disc it evolves: it migrates within the disc, accretes gas from the disc opening a gap, and inevitable increases in mass (Zhu, Hartmann, Nelson \& Gammie 2012; Hall, Forgan \& Rice 2017).

Previous studies (Stamatellos \& Whitworth 2009a,b; Kratter, Murray-Clay \& Youdin 2010; Zhu, Hartmann, Nelson \& Gammie 2012) suggest that the most likely outcome of disc fragmentation is brown dwarfs. Stamatellos \& Whitworth (2009a) performed an ensemble of 12 simulations of self-gravitating, fragmenting discs producing in total $\sim 100$ objects; 67 percent of these objects are brown dwarfs, 30 per cent are low-mass hydrogen-burning stars and only 3 per cent are planets. Additionally, they point out that the objects that end up as planets $\left(<13 M_{\mathrm{J}}\right)$, they are ejected from the disc quickly after their formation, thus avoiding any subsequent gas accretion. These ejected planets contribute to the suspected large population of free-floating planets. Stamatellos \& Whitworth (2009a) also argue that objects that remain in the disc, although they are planets when they form (i.e. their mass is below $\sim 13 M_{\mathrm{J}}$ ), they grow in mass to become either brown dwarfs or hydrogen-burning stars.

Another problem of the disc fragmentation theory is whether planets forming early on in protoplanetary discs are able to avoid fast inward migration towards their parent star. Previous studies suggest that the giant planets that form in relatively massive protoplanetary discs migrate inwards rapidly, on a time-scale reminiscent of Type I migration, without opening up a gap in the disc (Vorobyov \& Basu 2005, 2006; Baruteau, Meru \& Paardekooper 2011; Michael, Durisen \& Boley 2011; Malik et al. 2015). However, this has been questioned by the studies of Lin \& Papaloizou (2012) and Cloutier \& Lin (2013), which show that planets may migrate outwards due to gravitational edge mode instabilities. Stamatellos (2015) showed that planets are able to open gaps as they become more massive by accreting gas from the disc; by doing so, their inward migration slows down or even changes to outward migration.

An interesting variant of the gravitational fragmentation theory is the tidal downsizing hypothesis (see review by Nayakshin 2017a), in which clumps that form by disc fragmentation contract slowly while they migrate inwards. They may eventually get tidally disrupted, losing mass and possibly leaving behind a solid core. Nayakshin (2017b) finds that the final fate of a clump forming in the disc depends on how efficiently it cools and identifies two possible outcomes for the clump: it becomes either a brown dwarf on a wide orbit or a planet within 20 au from the central star. He concludes that even though disc fragmentation may commonly happen, only a small fraction of stars may have giant planets on wide orbits; this is corroborated by observations (Brandt et al. 2014; Galicher et al. 2016; Vigan et al. 2017) which show that only 1-10 per cent of star hosts gas giant planets on wide orbits.

Recent ALMA observations of the disc of the young star HL Tau (ALMA Partnership et al. 2015) have revealed the presence of multiple gaps at mm wavelengths. These gaps may be carved either by planets (Dipierro et al. 2015) or formed due to other processes (Takahashi \& Inutsuka 2014; Gonzalez et al. 2015; Takahashi et al. 2016b). HL Tau is an extremely young object (Greaves et al. 2008) that shows signs of outflows in the form of jets and infall from its parent cloud. Its disc is relatively massive (0.1-0.15 $\mathrm{M}_{\odot}$; Testi et al. 2015) and presumably is still being fed with gas by its ambient cloud. Such gaps have been observed in other discs e.g. in TW Hydra (Andrews et al. 2016) and in HD 163296 (Isella et al. 2016). Numerical simulations using resistive magnetohydrodynamics have suggested that discs form at an early stage during star formation and that they may be relatively massive in comparison to the mass of their host stars (Machida, Inutsuka \& Matsumoto 2010, 2011a,b; Machida, Inutsuka \& Matsumoto 2014). ALMA observations strongly support that discs exist from the Class 0 phase (Tobin et al. 2016). They appear to be relatively massive and extended, and to have spiral arms indicative of gravitational instabilities (Pérez et al. 2016; Tobin et al. 2016). The possible presence of planets in such young discs raises the exciting possibility that planets may form much faster than it has been previously thought, and therefore their early evolution, no matter how they have formed, will occur within a relatively massive disc.

In this paper, we study the evolution of a Jupiter-like planet-seed, referred to as the protoplanet, which finds itself within a protostellar disc that is massive enough for its self-gravity to be important for its evolution. The assumed disc is close to being gravitationally unstable, meaning that weak spiral arms may develop, but the disc does not fragment. We use the term protoplanet even though we refer to an object that only starts off as planet in the disc (irrespective of its formation mechanism). This object may accrete mass to eventually stop being a planet and become a brown dwarf $\left(m>13 M_{\mathrm{J}}\right)$. Our aim is to investigate whether such an object may actually survive as a planet by performing a set of numerical experiments. We expand on the work of Stamatellos (2015), exploring a wider parameter space. More specifically we investigate the effect of different disc viscosity, dust opacities, the orientation of the protoplanet's orbit, the protoplanet's initial orbital radius, and the effect of the radiation from the host star and the protoplanet itself. We therefore examine in detail how robust are the results of Stamatellos (2015). Additionally, we explore the reasons behind the different results with previous studies, comparing with simulations using a parametrized prescription for the disc cooling $(\beta$-cooling approximation).

We describe the initial condition of the simulations performed in Section 2, and in Section 3 the hydrodynamic method used and its ingredients (radiative transfer method, opacities, radiative feedback). We present the first set of simulations performed and their results in Section 4, commenting in detail on how fast the protoplanet grows in mass, and how its orbital properties (semimajor axis, eccentricity) change during its early evolution. In Section 5, we compare the results of our simulations with those of previous studies that employ the $\beta$-cooling approximation. In Section 6, we examine in detail the evolution of protoplanets at different radii from the central star. In Section 7, we present possibly the more realistic set of simulations, in which both radiative feedback from the planet and the host star are taken into account. Finally, in Section 8 we discuss the implications of this work for planet formation and evolution studies. 


\section{COMPUTATIONAL SETUP}

We assume a star with initial mass $M_{\star}=1 \mathrm{M}_{\odot}$ that is attended by a protostellar disc with mass $M_{\mathrm{D}}=0.1 \mathrm{M}_{\odot}$ and initial radius $R_{\mathrm{D}}=100 \mathrm{au}$. The disc is modelled by $10^{6}$ smoothed particle hydrodynamics (SPH) particles. The discs that we model are chosen so that they are not gravitationally unstable $(Q \gtrsim 1.5)$. Therefore, they are not expected to develop strong spiral arms (unless these are induced from the protoplanet).

The disc initial surface density is

$\Sigma_{0}(R)=\Sigma(1 \mathrm{au})\left(\frac{R}{\mathrm{au}}\right)^{-1}$,

and the disc temperature is

$T_{0}(R)=250 \mathrm{~K}\left(\frac{R}{\mathrm{au}}\right)^{-3 / 4}+10 \mathrm{~K}$,

where $\Sigma(1 \mathrm{au})$ is determined by the disc mass and radius, and $R$ is the distance from the central star measured on the disc mid-plane. The above density and temperature profiles are consistent with observations of late-phase (T Tauri) discs, but the properties of discs in the early phase are uncertain (MacFarlane \& Stamatellos 2017). Andrews et al. (2009) observed a sample of circumstellar discs in Ophiuchus and estimated a disc surface density that drops with the distance from the host star as $p \approx 0.4-1.0$. In Taurus-Auriga and Ophiuchus-Scorpius star formation regions, Andrews \& Williams (2007) find a median $p \approx 0.5$; they also find a temperature profile that drops with distance as $q \approx 0.4-0.74$. Osterloh \& Beckwith (1995) find that the disc temperature drops with radius, with an exponent $q \approx 0.35-0.8$. We note though that the profile defined in equation (2) is the initial disc temperature profile, but as the disc evolves it acquires a temperature profile self-consistently within the radiative hydrodynamic simulation.

The disc is allowed to relax, i.e. to evolve without a protoplanet, for three outer orbital periods ( $\sim 3 \mathrm{kyr})$. A protoplanet with a mass of $M_{\mathrm{p}}=1 M_{\mathrm{J}}$ is then embedded in the disc. The protoplanet's initial orbital velocity is set the same as the orbital velocity of the local gas, i.e. Keplerian (including the contribution from the disc mass within the radius of the protoplanet). We additionally assume an initially circular orbit $\left(e_{i}=0\right)$.

The choice of the initial disc mass $\left(0.1 \mathrm{M}_{\odot}\right)$ is intentionally conservative. If the protoplanet has formed by disc fragmentation then numerical studies indicate that a disc needs to have mass at least a few tenths $\mathrm{M}_{\odot}$ (e.g. the simulations of Stamatellos et al. 2011a suggest that a disc around a $0.7 \mathrm{M}_{\odot}$ star needs to be more massive than $0.25 \mathrm{M}_{\odot}$ in order to fragment).

The choice of the initial mass of the protoplanet $\left(1 M_{\mathrm{J}}\right)$ is also conservative. The minimum mass of an object forming by gravitational fragmentation is set by the thermodynamics of gas, as in order for a condensation to collapse, the energy delivered by compression needs to be efficiently radiated away. The energy loss of a fragment is determined by its opacity, so this is usually referred to as the opacity limit for fragmentation (e.g. Whitworth \& Stamatellos 2006; Whitworth et al. 2007). Theoretical studies estimate a minimum mass for fragmentation to be $1-10 M_{\mathrm{J}}$ (Low \& Lynden-Bell 1976; Rees 1976; Silk 1977; Boss 1988; Masunaga \& Inutsuka 1999; Boyd \& Whitworth 2005; Whitworth \& Stamatellos 2006; Boley et al. 2010; Kratter et al. 2010; Forgan \& Rice 2011; Rogers \& Wadsley 2012). We therefore study a system in which both the disc mass and the initial protoplanet mass are close to the lower limits that they may have, if the protoplanet has formed by disc fragmentation. However, our study is general and applies even for planets that have formed by core accretion.

\section{COMPUTATIONAL METHODS}

\subsection{Gas hydrodynamics and radiative transfer}

We use the SPH code SEREN (Hubber et al. 2011a,b) to treat the disc thermodynamics. The code uses an octal tree to compute gravity and find neighbours, multiple particle time-steps for optimization, and a second Runge-Kutta integration scheme. We use a timedependent artificial viscosity (Morris \& Monaghan 1997) with parameters $\alpha_{\min }=0.1, \alpha_{\max }=1$, and $\beta=2 \alpha$, so as to reduce artificial shear viscosity. The chemical and radiative processes that determine the disc temperature are treated with the diffusion approximation of Stamatellos et al. (2007) and Forgan et al. (2009). The net radiative heating rate for an SPH particle $i$ is

$\left.\frac{\mathrm{d} u_{i}}{\mathrm{~d} t}\right|_{\mathrm{RAD}}=\frac{4 \sigma_{\mathrm{SB}}\left(T_{\mathrm{A}}^{4}-T_{i}^{4}\right)}{\bar{\Sigma}_{i}^{2} \bar{\kappa}_{\mathrm{R}}\left(\rho_{i}, T_{i}\right)+\kappa_{\mathrm{P}}^{-1}\left(\rho_{i}, T_{i}\right)}$,

where $u_{i}$ is the specific internal energy of the particle, and $\rho_{i}, T_{i}$, its density and temperature, respectively. The positive term on the right-hand side represents heating by the various radiation sources (star, protoplanet, and pseudo-background radiation field; see Section 3.4), and ensures that the gas and dust cannot cool radiatively below the pseudo-background temperature $T_{\mathrm{A}} . \sigma_{\mathrm{SB}}$ is the StefanBoltzmann constant, $\bar{\Sigma}_{i}$ is the mass-weighted mean column-density, and $\bar{\kappa}_{\mathrm{R}}\left(\rho_{i}, T_{i}\right)$ and $\kappa_{\mathrm{P}}\left(\rho_{i}, T_{i}\right)$ are suitably adjusted Rosseland- and Planck-mean opacities (see Stamatellos et al. 2007, for details). The method takes into account compressional heating, viscous heating, heating by the background radiation field, and radiative cooling/heating. The method has previously applied to disc studies (Stamatellos \& Whitworth 2008, 2009b; Stamatellos et al. 2011a) and has given similar results with grid-based computational methods (Boley et al. 2006; Cai et al. 2008). The method used has been shown that may overestimate the column density through which the disc gas cools (Wilkins \& Clarke 2012; Young et al. 2012; Lombardi, McInally \& Faber 2015) by a factor of a few; however, considering that the dust opacity in discs is not known accurately enough, this limitation of the method is not critical for the results presented in this paper. The detailed treatment of radiative transfer is important when considering how the gas fragments but it is not expected to be crucial (at least qualitatively) for the issues relating to the mass growth and migration of protoplanets already formed in the disc that are discussed in this paper.

\subsection{Opacities}

We use two prescriptions for the opacity of the disc (see Fig. 1):

(i) The Bell \& Lin (1994) opacity that is parametrized as

$\kappa(\rho, T)=\kappa_{0} \rho^{l} T^{m}$,

where $\kappa_{0}, l, m$ are constants that depend on the species and the physical processes that contribute to the opacity at different densities and temperatures (note that Planck-mean and Rosseland-mean opacities are assumed to be the same). For the temperatures in the discs, we simulate here (up to $\sim 1000 \mathrm{~K}$ ) the opacity is due to dust grains that at low temperatures are coated with ice, which evaporates at higher temperatures. The dust grains start to evaporate at around $1000 \mathrm{~K}$.

(ii) The Semenov et al. (2003) model includes similar physical processes but the dust considered includes spherical and aggregate 


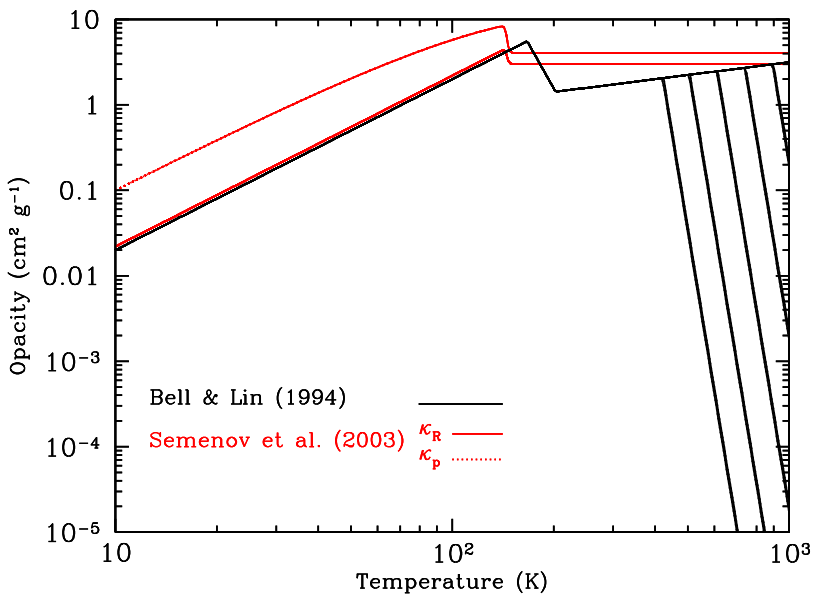

Figure 1. The two types of opacity used for the simulations presented in this paper: the Bell \& Lin (1994) opacities (black lines; different lines correspond to different densities), and the Semenov et al. (2003) opacities (red lines) that have been proposed for protostellar discs.

particles of various sizes that consist of ice, organics, troilite, silicates, and iron. This composition is thought to be more appropriate for the conditions found in protoplanetary discs.

The Semenov et al. (2003) Rosseland-mean opacity is similar to the Bell \& Lin (1994) one at temperatures up to $~ 100 \mathrm{~K}$ but higher at larger temperatures by up to a factor of 2 . At such temperatures the optically thick parts of the disc cool less efficiently when using the Semenov et al. (2003) opacity. The Semenov et al. (2003) Planckmean opacity is larger by a factor of 5 than the Bell \& Lin (1994) one, which means that the optically thin parts of the disc cool more efficiently.

The above two sets of opacities are widely used in the literature. However, the composition of dust in discs is rather uncertain, especially since significant dust growth may occur, lowering the opacity. It is unclear whether dust growth is significant at the early disc phase that we study here.

\subsection{Sinks}

Sink particles are used to represent the central star and the protoplanet (Bate, Bonnell \& Price 1995). Sink particles interact with the rest of the computational domain only through their gravity (and their luminosity if their radiative feedback in considered; see below). The sink radius of the central star is $R_{\text {sink, } \star}=0.2$ au, and the sink radius of the protoplanets is $R_{\text {sink, } \mathrm{p}}=0.1$ au. This value is chosen to be smaller than the Hill radius of the protoplanet, i.e. the region around it where its gravity dominates over the gravity of the star:

$R_{\text {sink, }}<R_{\mathrm{H}}=R\left(\frac{M_{\mathrm{p}}}{3 M_{\star}}\right)^{1 / 3}$.

For example, when a Jovian planet is at 50 au away from the central star, then its Hill radius is $R_{\mathrm{H}} \sim 3.5 \mathrm{au}$. The Hill radius may increase as the protoplanet accretes material from the disc or it may decrease as the protoplanet moves closer to the central star.

Gas particles accrete on to a sink when they are within the sink radius and bound to the sink (Hubber et al. 2011b). The accretion rate on to the sink is computed by calculating the increase of the sink mass over a time interval that is comparable to the dynamical time of each particle at the edge of the sink.

\subsection{Radiative feedback from the protoplanet and the star}

The radiation feedback from the protoplanet is taken into account (in a subset of the simulations) using the method described in Stamatellos (2015). We use the method of Stamatellos, Whitworth \& Hubber (2011b) \& Stamatellos, Whitworth \& Hubber (2012), which invoke a pseudo-ambient radiation field with temperature $T_{\mathrm{A}}^{\text {planet }}(\boldsymbol{r})$ due to radiation from the protoplanet (see also Stamatellos et al. 2007; Stamatellos \& Whitworth 2009a). This pseudo-ambient temperature sets the minimum temperature that the gas can attain if it cools radiatively. The contribution from the protoplanet is set to

$T_{\mathrm{A}}^{\text {planet }}(\boldsymbol{r})=\left(\frac{L_{p}}{16 \pi \sigma_{\mathrm{SB}}\left|\boldsymbol{r}-\boldsymbol{r}_{\mathrm{p}}\right|^{2}}\right)^{1 / 4}$,

where $\boldsymbol{r}$ is the position on the disc mid-plane, and $L_{\mathrm{p}}$ and $\boldsymbol{r}_{\mathrm{p}}$ are the luminosity and position of the protoplanet, respectively. The above approximation may overestimate the effect of the feedback in optically thick regions of the disc, which are well shielded from the protoplanet (see discussion in Mercer \& Stamatellos 2017).

The luminosity of the protoplanet is set to

$L_{\mathrm{p}}=f \frac{G M_{\mathrm{p}} \dot{M}_{\mathrm{p}}}{R_{\mathrm{acc}}}$

where $M_{\mathrm{p}}$ is the mass of the protoplanet, $\dot{M}_{\mathrm{p}}$ is the accretion rate on to it, and $R_{\text {acc }}$ is the assumed accretion radius. $f=0.75$ is the fraction of the accretion energy that is radiated away at the photosphere of the protoplanet, rather than being expended driving jets (Machida, Inutsuka \& Matsumoto 2006; Offner et al. 2009), or deposited within the protoplanet (e.g. Baraffe et al. 2017).

The exact amount of the energy radiated away from the protoplanet depends on the detailed properties of the accretion shock around the protoplanet (Zhu 2015; Marleau et al. 2017; Mordasini, Marleau \& Mollière 2017; Szulágyi \& Mordasini 2017). If this protoplanet has formed by gravitational instabilities in the disc, then the accretion happens on to the second hydrostatic core (e.g. Larson 1969; Stamatellos \& Whitworth 2009b). The radius of the second core is uncertain: $\sim 1-20 \mathrm{R}_{\odot}$ (Masunaga \& Inutsuka 2000; Tomida et al. 2013; Vaytet et al. 2013; Bate 2014; Tsukamoto et al. 2015). Here, we assume $R_{\text {acc }}=3 \mathrm{R} \odot$, but the results of this work are not sensitive on this assumption. The accretion luminosity of the protoplanet is significant due to the relatively high accretion rate on to it during the initial stages of its evolution and can dominate over the stellar luminosity in the disc region around the protoplanet (Owen 2014; Montesinos et al. 2015; Stamatellos 2015), especially for the Jupiter-like planet-seeds in high-mass discs that we study here.

If the protoplanet has formed by disc fragmentation, on top of the significant accretion luminosity, it will also radiate energy due to its contraction. This energy release is of the order of $0.1 \mathrm{~L} \odot$ (Inutsuka, Machida \& Matsumoto 2010), which is similar to the one expected from accretion. Therefore, the protoplanet's luminosity is deemed to play an important role in its evolution (Nayakshin \& Cha 2013; Benítez-Llambay et al. 2015; Stamatellos 2015). We do not take this radiation explicitly into account in the simulations presented in this paper, but we have performed runs with smaller $R_{\text {acc }}$ resulting in higher luminosity for the protoplanet. These runs give qualitatively similar results to the ones we discuss in this paper.

The radiation feedback from the star is taken into account (in a subset of the simulations) by invoking an additional pseudo-ambient temperature,

$T_{\mathrm{A}}^{\star}(\boldsymbol{r})=T(1 \mathrm{au})\left(\frac{R}{\mathrm{au}}\right)^{-3 / 4}+10 \mathrm{~K}$, 
where $R$ is the distance from the central star on the disc mid-plane, and $T_{0}$ is the temperature at $R=1$ au from the star. The total pseudoambient temperature (due to the central star and the protoplanet) is then set to

$T_{\mathrm{A}}^{4}(\boldsymbol{r})=\left[T_{\mathrm{A}}^{\star}(\boldsymbol{r})\right]^{4}+\left[T_{\mathrm{A}}^{\text {planet }}(\boldsymbol{r})\right]^{4}$,

where $T_{\mathrm{A}}^{\text {planet }}(\boldsymbol{r})$ is the contribution from the protoplanet. Note that the radiative feedback from the star is fixed in time for simplicity, whereas the radiative feedback from the protoplanet is variable and depends on the accretion rate of gas on to it as it moves within the disc.

\section{SIMULATIONS OF PROTOTOPLANETS EVOLVING IN YOUNG DISCS}

We follow the evolution of a protoplanet embedded in the disc for $20 \mathrm{kyr}$ (approximately 20 disc outer orbital periods). The initial mass of the protoplanet is $M_{p, i}=1 M_{\mathrm{J}}$ and it is placed at distance $R_{p, i}=50 \mathrm{au}$. In this first set of simulations, we ignore any radiative feedback from the central star. The effect of irradiation from the central star will be studied in Sections 7 and in Appendix B. We perform the following five numerical experiments (see Table 1):

(i) Run 1: no radiative feedback from the central star nor the protoplanet are included (hereafter referred to as the standard run).

(ii) Run 2: radiative feedback from the protoplanet is included.

(iii) Run 3: same as the standard run but with higher viscosity (viscosity $\alpha_{\min }=0.2 ; \alpha_{\min }=0.1$ in all other runs).

(iv) Run 4: same as the standard run but in which the protoplanet has an orbit that has an initial inclination of $10^{\circ}$ with respect to the disc mid-plane.

(v) Run 5: same as the standard run but using different opacities: Semenov et al. (2003); for all other runs we use the Bell \& Lin (1994) opacities (see Section 3.2).

The evolution of the disc surface density for all five runs is shown in Fig. 2. We calculate the migration time-scale as

$\tau_{\text {mig }}=\alpha_{\mathrm{p}} /\left|\dot{\alpha}_{\mathrm{p}}\right|$,

where $\alpha_{\mathrm{p}}$ is the protoplanet's semimajor axis. In all runs the protoplanet initially migrates inwards fast (see Tables A1 and A2), with a migration time-scale of $\sim 10^{4} \mathrm{yr}$, as previous studies have found (Vorobyov \& Basu 2006; Baruteau et al. 2011; Michael et al. 2011; Zhu et al. 2012; Malik et al. 2015). However, as the protoplanet migrates inwards it considerably grows in mass by accreting gas from the disc. Eventually the protoplanet is able to open up a gap and the migration slows down (for the case with radiative feedback from the protoplanet; migration time-scale $\sim 10^{5} \mathrm{yr}$ ) or even changes to

Table 1. Simulation parameters.

\begin{tabular}{lcccccc}
\hline Run id & Opacity & $\alpha_{\text {min }}^{a}$ & $\begin{array}{c}\alpha_{p, i}^{b} \\
(\mathrm{au})\end{array}$ & $\begin{array}{c}M_{p, f}^{c} \\
\left(M_{\mathrm{J}}\right)\end{array}$ & $\begin{array}{c}\alpha_{p, f}^{d} \\
(\mathrm{au})\end{array}$ & $e_{f}^{e}$ \\
\hline Run 1 & BL94 $^{f}$ & 0.1 & 40 & 24 & 40 & 0.035 \\
Run 2 & BL94 $^{f}$ & 0.1 & 40 & 15 & 17 & 0.0012 \\
Run 3 & BL94 $^{f}$ & 0.2 & 40 & 29 & 37 & 0.13 \\
Run 4 & BL94 $^{f}$ & 0.1 & 40 & 25 & 36 & 0.017 \\
Run 5 & SEM03 $^{g}$ & 0.1 & 40 & 31 & 53 & 0.17 \\
\hline
\end{tabular}

Notes. ${ }^{a}$ Minimum SPH artificial viscosity. ${ }^{b}$ Initial semimajor axis of the protoplanet. ${ }^{c}$ Final protoplanet mass. ${ }^{d}$ Final semimajor axis of the protoplanet. ${ }^{e}$ Final protoplanet eccentricity. ${ }^{f}$ Bell \& Lin (1994) opacity. ${ }^{g}$ Semenov et al. (2003) opacity. an outward direction (migration time-scale $\sim 10^{5} \mathrm{yr}$ ) in the rest of the runs (see Stamatellos 2015). After a gap is opened up, the protoplanet grows in mass slowly, by accreting a significant amount of material from the disc outside its orbit. An important difference of this study from previous ones (e.g. Baruteau et al. 2011; Michael et al. 2011) is that the protoplanet is allowed to grow in mass. This is especially significant for the case of self-gravitating discs as the protoplanet evolves in a relatively massive disc with a significant amount of gas available for accretion. In the following subsections, we discuss in detail the evolution of the protoplanet within the disc in the different runs we have performed.

\subsection{Protoplanet mass growth}

Initially, the protoplanet grows very fast in mass, as it opens up a gap but its mass increase slows down once the gap is opened up (see Fig. 3). The accretion rate on to the protoplanet (see Fig. 4, top) is relatively high $\left(\sim 10^{-4}-10^{-2} M_{\mathrm{J}} \mathrm{yr}^{-1}\right)$ during the gap opening phase but then it drops down; nevertheless, accretion continues through streams within the gap (Lubow \& D'Angelo 2006). Similarly high accretion rates are also seen in previous studies (D'Angelo \& Lubow 2008; Ayliffe \& Bate 2009; Zhu et al. 2012; Gressel et al. 2013). The resulting luminosity of the protoplanet (see Fig. 4, bottom) could rival the luminosity of the young star making its detection easier in terms of the sensitivity required. However, this phase of high accretion lasts only for a relatively short time, making such a detection difficult. These luminosity estimates are only up to a few times higher than the luminosities estimated from hot-start models of planet formation (Marley et al. 2007; Mordasini 2013; Mordasini et al. 2017). The value of the luminosity depends on the accretion radius, which in our model is set assuming that gas accretion happens on to the second core formed after the temperature at centre of the protoplanet-precursor clump reaches $\sim 2000 \mathrm{~K}$ and molecular hydrogen dissociates (see e.g. Stamatellos \& Whitworth 2009 b). The accretion rate on to the protoplanet decreases after the gap is opened up but thereafter it shows many spikes, some with periodicity similar to the orbital period of the planet at each specific time, indicating periodic interactions with spiral structures in the disc that drive gas accretion on to the protoplanet. Such spikes are absent when the radiative feedback of the planet is taken into account (Run 2) as strong spiral features are suppressed. There is a delay of $\sim 2-4$ kyr between the gap opening and the gap edges becoming gravitationally unstable, driving accretion on to the protoplanet. The delay is longer for protoplanets that are closer to the central star, i.e. in a warmer region of the disc, as more gas needs to accumulate for the gap edges to become unstable. The accretion rate on to the protoplanet is generally lower when the protoplanet's radiative feedback is included in the simulation as seen in previous studies (Nayakshin \& Cha 2013; Stamatellos 2015; Gárate, Cuadra \& Montesinos 2017).

The way that gas is accreted on to the protoplanet depends on the dynamical state of the protostellar disc. In Fig. 5, we plot the difference between the angular momentum of the gas entering the protoplanet's Hill sphere from inside the protoplanet's orbit $\left(L_{\text {in }}\right)$, and the angular momentum of the gas entering the Hill sphere from outside the protoplanet's orbit $\left(L_{\text {out }}\right)$. To calculate these, we use the mass of gas within distance $1<r<1.2 R_{\mathrm{H}}{ }^{1}$ from the protoplanet that moves towards the protoplanet from inside its orbit $\left(m_{\text {in }}\right)$, and

\footnotetext{
${ }^{1}$ The same result is obtained for a different outer limit, e.g. if we consider the gas within distance $1<r<1.5 R_{\mathrm{H}}$ from the protoplanet.
} 


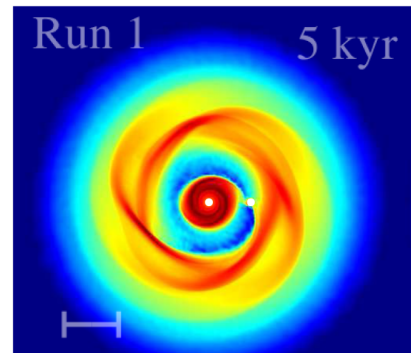

$50 \mathrm{AU}$

Run 2

\section{(-)}

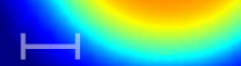

$50 \mathrm{AU}$

Run 3

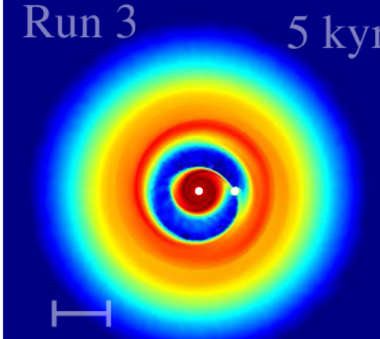

$50 \mathrm{AU}$

Run 4

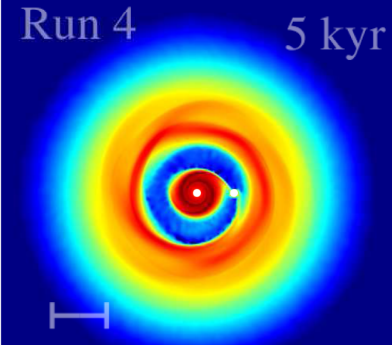

$50 \mathrm{AU}$

Run 5

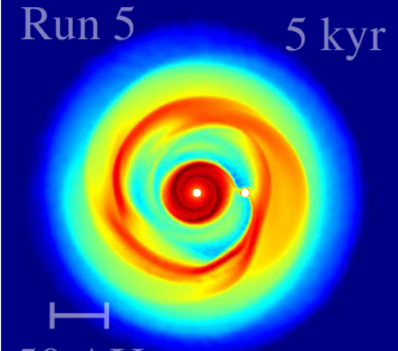

$50 \mathrm{AU}$

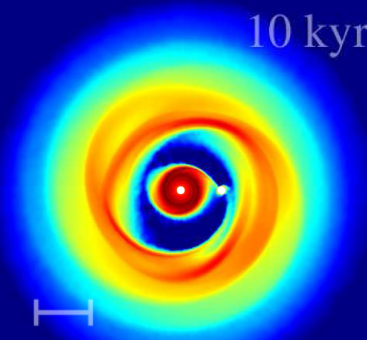

$50 \mathrm{AU}$

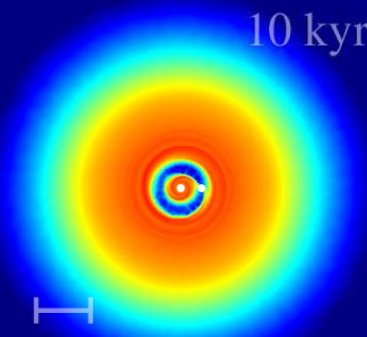

$50 \mathrm{AU}$

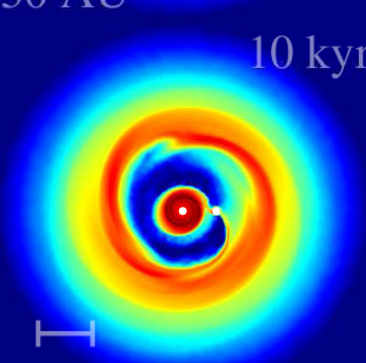

$50 \mathrm{AU}$

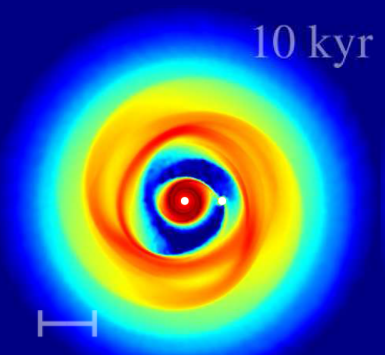

$50 \mathrm{AU}$

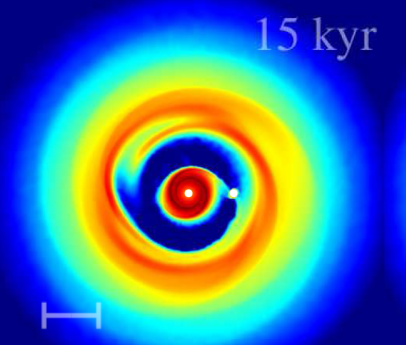

$50 \mathrm{AU}$

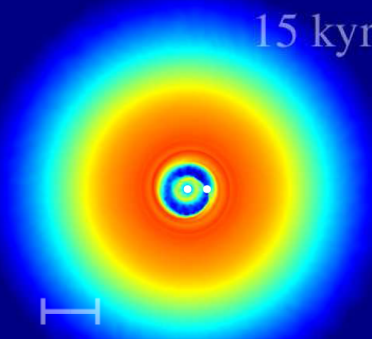

$50 \mathrm{AU}$

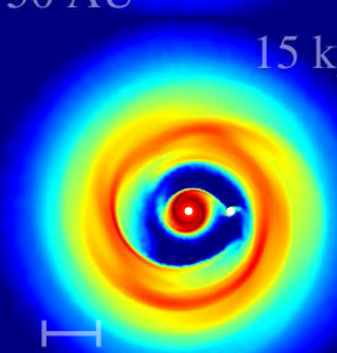

$50 \mathrm{AU}$

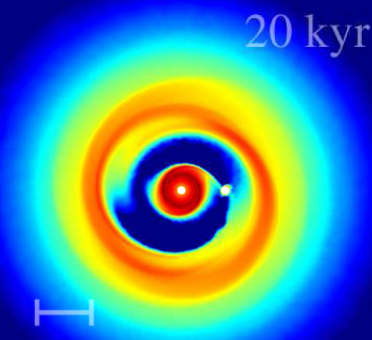

$50 \mathrm{AU}$

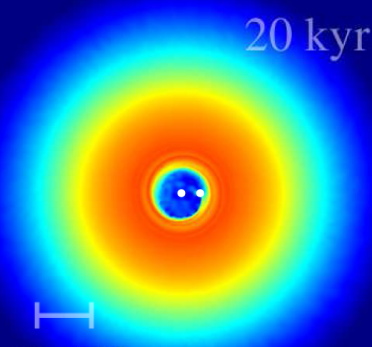

$50 \mathrm{AU}$

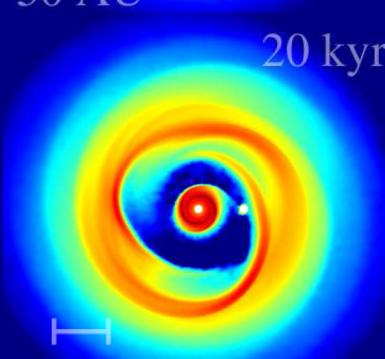

$50 \mathrm{AU}$

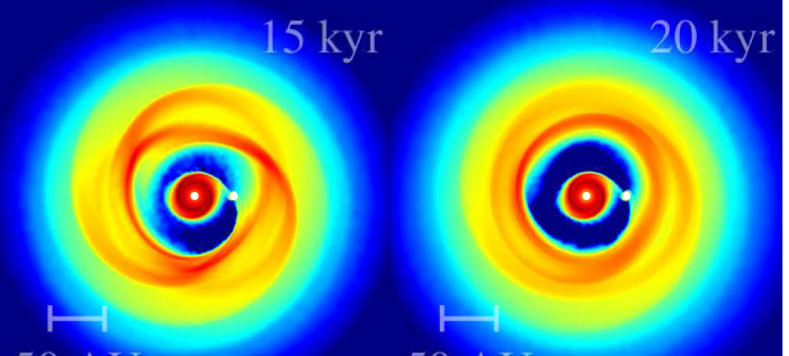

$50 \mathrm{AU}$
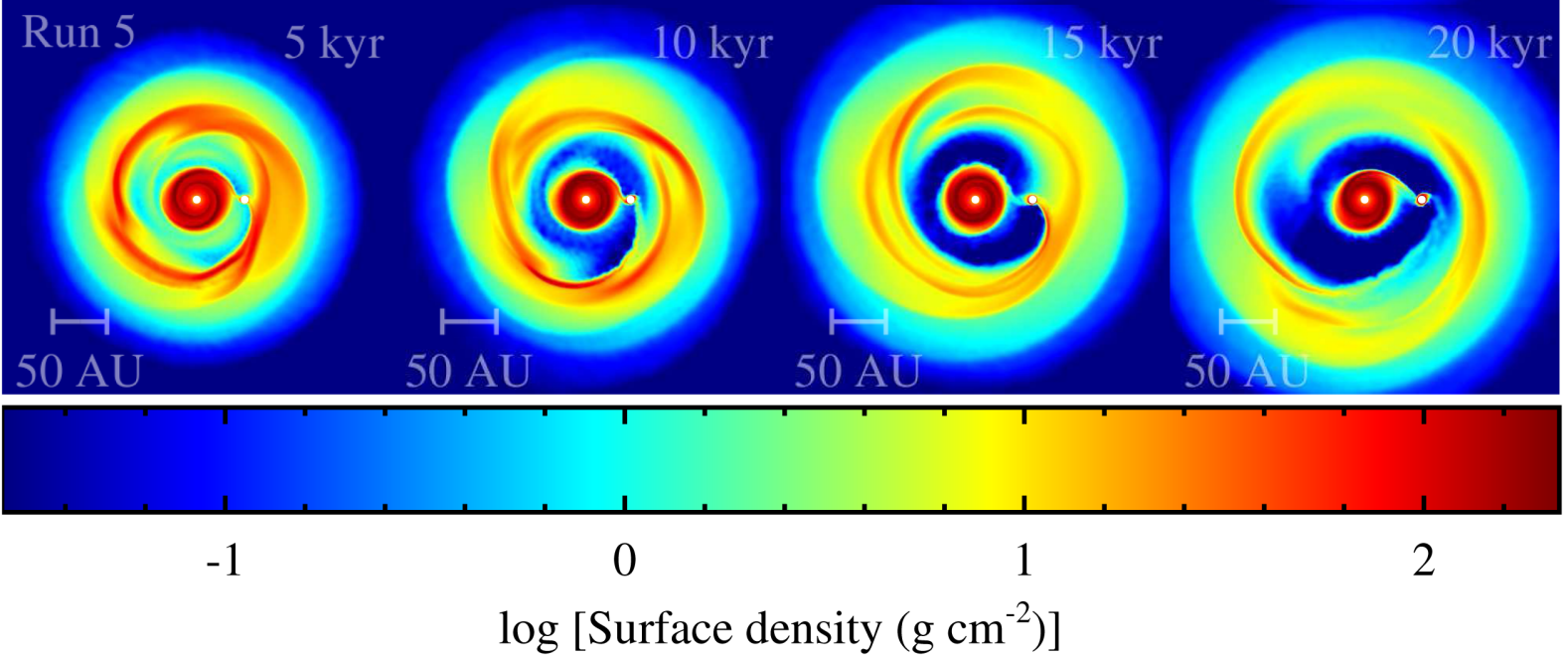

Figure 2. The evolution of the disc surface density in the five simulations listed in Table 1. The star and the protoplanet as depicted as wide dots. Each row corresponds to different snapshots of each run at times as stated on the graph. All runs show similar features, apart from the run in which the radiative feedback of the protoplanet is taken into account (Run 2; second row). 


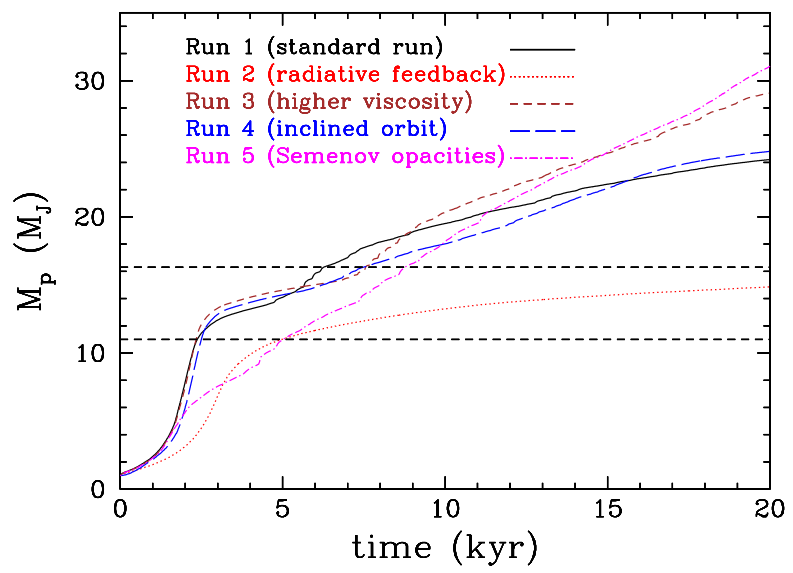

Figure 3. The evolution of the mass $M_{\mathrm{p}}$ of the protoplanet for the runs listed in Table 1. The increase in the protoplanet's mass is initially rapid as it opens up a gap and in most cases by the end of the simulation it has become a brown dwarf ( $m>11-16.3 M_{\mathrm{J}}$; Spiegel, Burrows \& Milsom 2011, these limits are depicted by the horizontal dashed lines). When the radiative feedback of the protoplanet is taken into account (Run 2), its mass growth is suppressed and its mass remains within the planetary-mass regime.

the gas that moves towards the protoplanet from outside its orbit $\left(m_{\text {out }}\right)$ (Fig. 5, bottom). During the initial gap opening phase, in both cases, gas is accreted on to the protoplanet mostly from the inner disc. After the initial gap opening phase ( $\sim 5 \mathrm{kyr})$, we see that for the run including the protoplanet's radiative feedback (Run 2, red), in which the disc is gravitationally stable because of the effect of the protoplanet's feedback, most of the accreted gas comes from inside the protoplanet's orbit. The total accreted gas in this case has lower angular momentum than the protoplanet, so that the protoplanet's angular momentum decreases. On the other hand, in the case without the feedback (Run 1, black), the disc is gravitationally unstable and a high fraction of the accreted gas (up to almost 50 per cent) comes from outside the protoplanet's orbit. This gas has higher angular momentum than the protoplanet.

This can also be seen in Fig. 6 where we plot the change of angular momentum (top) and mass (bottom) of the protoplanet ( $\Delta L_{\mathrm{p}}$, $\Delta M_{\mathrm{p}}$ ), the inner disc (i.e. the disc inside the protoplanet's orbit; $\Delta L_{\text {inner disc }}, \Delta M_{\text {inner disc }}$ ), and the outer disc ( $\left.\Delta L_{\text {outer disc }}, \Delta M_{\text {outer disc }}\right)$. In Run 1, the protoplanet increases in mass while the outer disc mass decreases, whereas in Run 2, in which the protoplanet's feedback is included, the outer disc mass remains almost steady (after $5 \mathrm{kyr}$ ), whereas the inner disc mass decreases as the mass of the protoplanet increases.

Apart from the run with the protoplanet's radiative feedback, there are only relatively subtle differences in the accretion on to the protoplanets in the other runs. As expected, for a higher disc viscosity the gap opens up at a later time (Crida, Morbidelli \& Masset 2006), but thereafter the accretion rate into the protoplanet is higher, resulting in a higher final mass.

The gap opens up faster in the run with the Semenov et al. (2003) opacities, which are generally larger than the Bell \& Lin (1994) opacities, and the accretion rate thereafter is larger by a factor of $\sim 2$ than in the other runs. This is because the Semenov et al. (2003) opacities correspond to more efficient cooling (see Nayakshin 2017b), and therefore (i) the disc is cooler, enabling the quick opening of the gap, and (ii) the thermal pressure of the
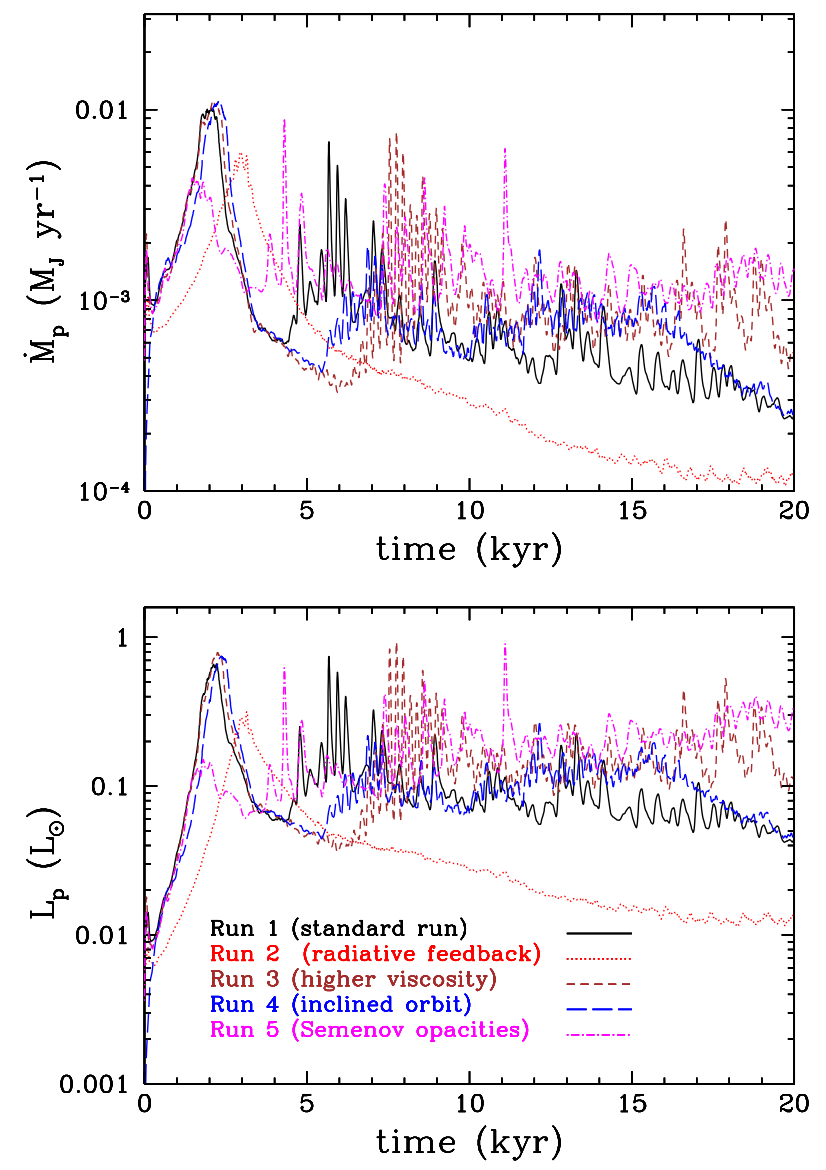

Figure 4. The mass accretion on to the protoplanet (top) and the resulting accretion luminosity (bottom). The accretion rate on to the protoplanet is high, and therefore the protoplanet's luminosity may rival that of its host star, but probably only for a short period of time. (Note that in all runs in this set, apart from Run 2, this luminosity is not fed back into the disc.)

circumplanetary disc is lower and gas accretion is not opposed as much as in the other runs. The dependence of the accretion rate and the final mass of the object on the assumed opacity seems to be different from what is seen in Nayakshin (2017b). However, what matters is not the opacity itself but the cooling of the gas around the protoplanet. Nayakshin (2017b) employs an opacity that regulates gas cooling both in optically thin and optically thick regimes (i.e. the Planck-mean and Rosseland-mean opacities are the same), whereas the Semenov et al. (2003) opacity account of the differences between these regime. The higher Planck-mean opacity of Semenov et al. (2003) results in more efficient cooling in the optically thin regions (see equation 3 ; in the optically thin limit the denominator on the right-hand side is dominated by the $\kappa_{P}^{-1}$ term). Therefore, as in Nayakshin (2017b), our results mean that the gas accretion on to the protoplanet with more efficient cooling is higher. Similar results are also found by Ormel, Kuiper \& Shi (2015a) and Ormel, Shi \& Kuiper (2015b) when studying accretion on to massive solid planet cores.

We see that in all cases, apart from the one in which the protoplanet radiative feedback has been included, the protoplanet's final mass (i.e. the mass at the end of the hydrodynamic simulation; $t=20 \mathrm{kyr})$ is in the brown dwarf regime $\left(>16.3 \mathrm{M}_{\mathrm{J}}\right)$. The protoplanet's mass in the case in which its radiative feedback is taken into account is at the borderline between the planetary and brown dwarf regime. However, it is expected that the protoplanet will continue 


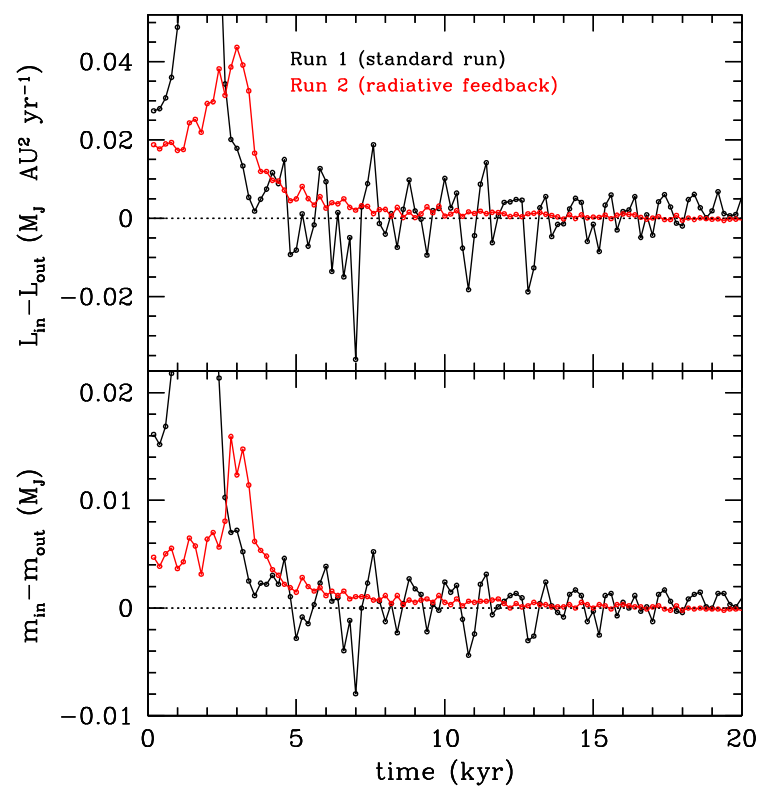

Figure 5. The difference between the mass of gas, within distance 1 to $1.2 R_{\mathrm{H}}$ from the protoplanet, which moves towards the protoplanet from inside its orbit $\left(m_{\text {in }}\right)$ and from outside its orbit $\left(m_{\text {out }}\right)$ (bottom) and the related difference between their angular momenta $\left(L_{\text {in }}-L_{\text {out }}\right)$. In the run including the protoplanets radiative feedback (Run 2, red), most of the accreted mass comes from inside the protoplanet's orbit, whereas in the case without this feedback (Run 1, black) an almost equal amount of accreted mass comes from outside the protoplanet's orbit. The average angular momentum difference is positive for the radiative feedback run (for $t>5 \mathrm{kyr}$ ), but negative for the standard (non-radiative feedback) run. After the gap opens up in the disc, in the former case the protoplanet loses angular momentum and migrate inwards, whereas in the latter case it gains angular momentum and migrates outwards, while accreting higher angular momentum gas.

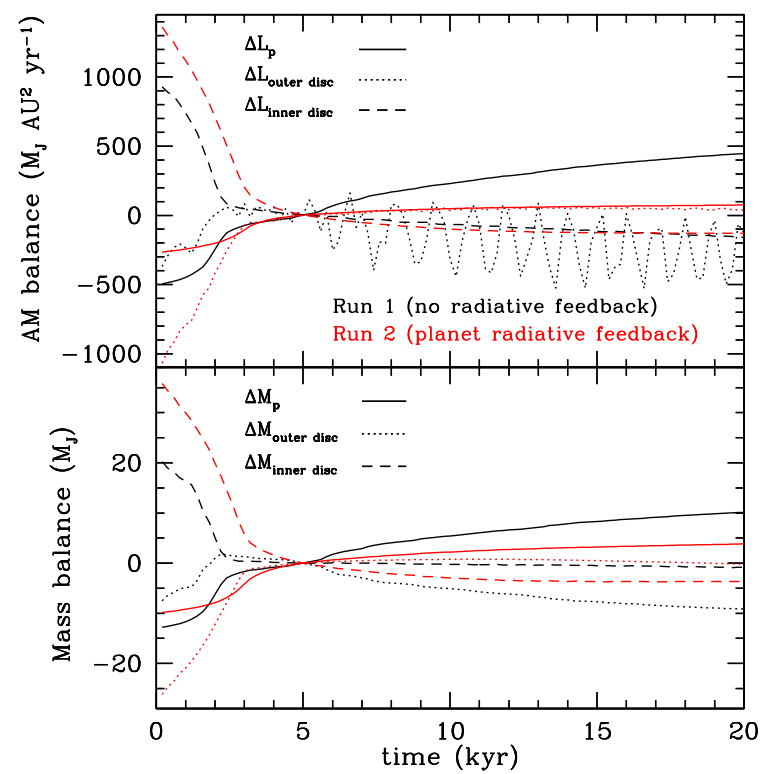

Figure 6. The change of angular momentum (top) and mass (bottom) of the planet $\left(\Delta L_{\mathrm{p}}, \Delta M_{\mathrm{p}}\right)$, the inner disc (i.e. the disc inside the protoplanet's orbit; $\Delta L_{\text {inner disc }}, \Delta M_{\text {inner disc }}$ ), and the outer disc ( $\left.\Delta L_{\text {outer disc }}, \Delta M_{\text {outer disc }}\right)$. The reference point is chosen at $t=5 \mathrm{kyr}$, so as to separate the gap opening phase from the subsequent slow migration phase.

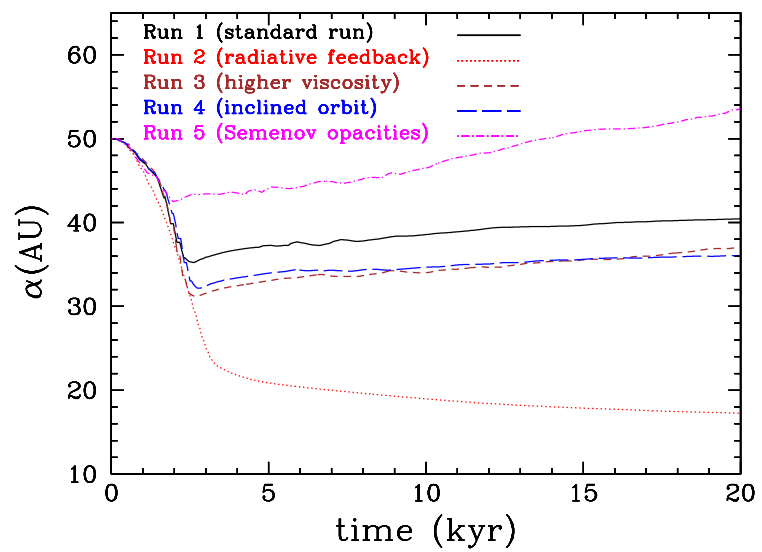

Figure 7. The semimajor axis evolution of an initially $1-M_{\mathrm{J}}$ protoplanet in a $0.1-\mathrm{M}_{\odot}$ disc. The protoplanet initially migrates inwards on a Type I migration time-scale $\left(\sim 10^{4} \mathrm{yr}\right)$ but once a gap opens up, the inward migration stops and turns into an outward migration, except for the case when the radiative feedback of protoplanet is taken into account. In the latter case, the migration continues inwards but at a Type II time-scale $\left(\sim 10^{5} \mathrm{yr}\right)$. (The details of the runs are listed in Table 1.)

to accrete gas and eventually become a brown dwarf (unless the disc dissipates by other processes, like e.g. photoevaporation, on a faster time-scale). Nevertheless, when the protoplanet's radiative feedback is taken into account the mass growth of the protoplanet has been suppressed and, at the end of the simulation, its mass is lower by a factor of $\sim 2$ than in the other runs.

\subsection{Protoplanet migration}

The protoplanet initially interacts strongly with the disc resulting in fast inward migration, similar to the Type I migration for low-mass planets in low-mass discs. The migration time-scale is only $10^{4} \mathrm{yr}$ and it is similar for all five runs (see Figs 7, 8 and Tables A1, A2). However, once a gap opens up the migration stalls, this happens at different times for each run. When the planet orbit plane is not the same with the disc mid-plane (Run 3), it is more difficult for the protoplanet to open up a gap. In the same way, the more viscous is the disc (Run 4) the more difficult is to open up a gap (e.g. Crida et al. 2006; Malik et al. 2015). In both cases, there is delay in opening up a gap and the protoplanet migrates further inwards (but only by $\sim 4$ au compared with the other runs). In the four runs without radiative feedback from the protoplanet, the protoplanet switches to a slow outward migration. At the end of the simulations (20 kyr), the protoplanet's semimajor axis has moved to 40, 38, and 36 au, for Runs 1, 2, and 3, respectively, from an initial semimajor axis of 50 au. In Run 5 with the higher opacity (faster cooling; Semenov et al. 2003), the gap opens up quicker and the protoplanet starts migrating outwards reaching a semimajor axis of 53 au at the end of the simulation.

The case that it is distinctly different is the one in which the radiative feedback from the planet is taken into account (Run 2). In this case, the opening up of the gap is more difficult as the protoplanet heats its circumplanetary disc but also the host protostellar disc (Marley et al. 2007; Nayakshin \& Cha 2013; Benítez-Llambay et al. 2015; Montesinos et al. 2015; Stamatellos 2015; Szulágyi \& Mordasini 2017). Therefore, the gap opens at a later time $(\sim 1 \mathrm{kyr}$ later) and the protoplanet migrates closer to central star. Its inward migration continues after the gap is opened up but at a much longer time-scale $\left(\sim 10^{5} \mathrm{yr}\right.$; typical of Type II migration). At the end of the 


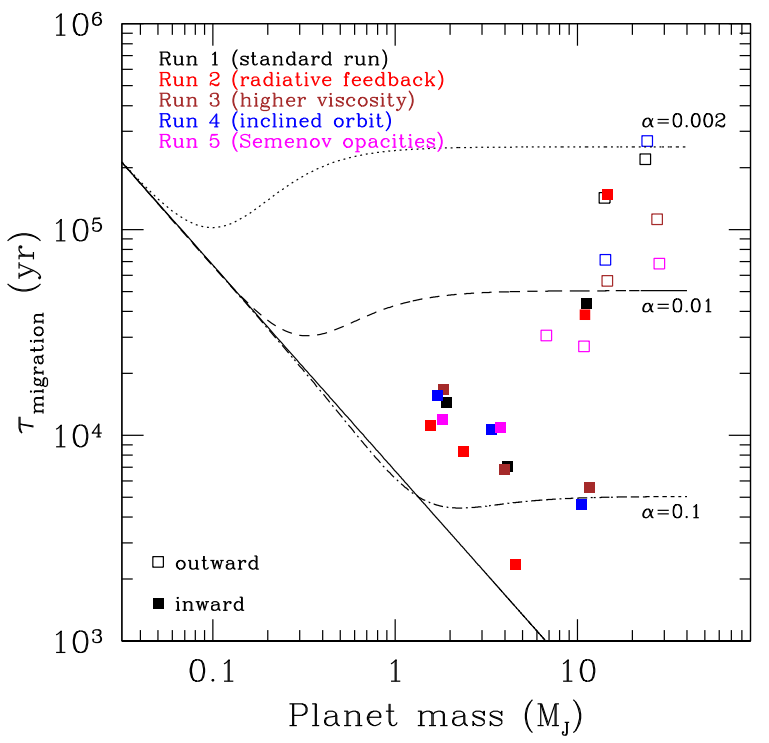

Figure 8. The migration time-scales at different times during the runs listed in Table 1. Filled boxes correspond to inward migration and empty boxes to outward migration. The solid line corresponds to the Type I migration time-scale, whereas the dashed lines correspond to a combination of Type I and Type II migration assuming different disc viscosity parameter $\alpha$ (see the text for details). Once the gap is opened up, interactions of the protoplanet with the gravitationally unstable gap edges lead to outward migration. When the radiative feedback from the planet is taken into account the gap edges are hotter, and therefore they are stable and the inward migration continues but at a longer time-scale.

simulations (20 kyr), the protoplanet is at 18 au from the central star.

Lin \& Papaloizou (2012) and Cloutier \& Lin (2013) have shown that when a planet resides in a gap that has gravitationally unstable edges, gas is brought into the planet's co-orbital region by the spiral arms, resulting in a positive torque that pushes the planet outwards. This is indeed what we see here in the runs without the radiative feedback from the protoplanet. In these runs, a significant amount of the gas accreted on to the protoplanet is high angular momentum material coming from outside the protoplanet's orbit (see Figs 5 and 6). On the other hand, when the radiative feedback from the protoplanet is taken into account the temperature at the edges of the gap is raised, they are not gravitationally unstable anymore, and the protoplanet accretes a higher fraction of lower angular momentum gas from the disc inside its orbit.

Contrary to previous studies of Baruteau et al. (2011), Michael et al. (2011), and Malik et al. (2015), we allow the protoplanet to accrete gas and grow in mass. By growing in mass, it is more capable to open up a gap. In previous studies, mass is accumulated in the circumplanetary disc and therefore contributed to strongly couple the protoplanet with the gaseous disc in which it is formed. This behaviour is also seen in the simulations of Nayakshin \& Cha (2013). However, the crucial difference with previous studies is that we use a more detailed prescription for the radiative transfer in the disc. Our prescription allows the circumstellar and circumplanetary material to moderate the efficiency of its cooling as it becomes more or less dense, whereas the studies of Baruteau et al. (2011) and Malik et al. (2015) use the $\beta$-cooling approximation, in which the cooling time is proportional to the local orbital period of the gas, i.e. $t_{\text {cool }}=\beta \Omega_{K}^{-1}(R)$. In Section 5, we discuss this issue in detail.
The estimated migration time-scales $\left(\tau_{\text {mig }}=\alpha_{\mathrm{p}} /\left|\dot{\alpha}_{\mathrm{p}}\right|\right)$ are shown in Table A1, and the migration rates $\left(v_{\text {mig }}=\alpha_{p}\right)$ in Table A2. These values are calculated at five different times during the simulations. The migration time-scales for all five runs are plotted against the protoplanet mass in Fig. 8. On the same graph, we plot the estimated migration time-scales for Type I and Type II migration as calculated by Ward (1997) and (Tanaka, Takeuchi \& Ward 2002, see also Bate et al. 2003).

The Type I migration rate estimated by Tanaka et al. (2002) is

$v_{I}=-\left(2.7+1.1 \alpha_{\Sigma}\right) \frac{M_{\mathrm{p}}}{M_{\star}} \frac{R_{\mathrm{p}}^{2} \Sigma_{\mathrm{p}}}{M_{\star}}\left(\frac{H}{R_{\mathrm{p}}}\right)^{-2} R_{\mathrm{p}} \Omega_{\mathrm{p}}$,

where $\alpha_{\Sigma}$ is the exponent of the disc surface density profile ( $\Sigma \propto$ $\left.R^{-\alpha_{\Sigma}}\right), R_{\mathrm{p}}$ is the orbital radius of the planet, $\Sigma_{\mathrm{p}}$ is the surface density of the disc at the position of the planet, $H_{\mathrm{p}}$ is the disc thickness at the position of the planet, and $\Omega_{\mathrm{p}}$ is the planet's angular frequency. The rate of migration of the Type II case is set by the viscous evolution of the disc (e.g. Bate et al. 2003):

$v_{I I}=-\frac{3}{2} \alpha\left(\frac{H_{\mathrm{p}}}{R_{\mathrm{p}}}\right)^{2} R_{\mathrm{p}} \Omega_{\mathrm{p}}$,

where $\alpha$ is the disc viscosity parameter. We note though that the actual Type II migration time-scale may vary from the above approximation (Crida \& Morbidelli 2007; Duffell et al. 2014; Dürmann \& Kley 2015, 2017). For example, Dürmann \& Kley $(2015,2017)$ find that due to gas crossing through the gap Type II migration may be faster or slower. The total rate of migration, combining the Type I and Type II regimes, is therefore

$v=\frac{v_{I}}{1+\left(M_{\mathrm{p}} / M_{\mathrm{t}}\right)^{3}}+\frac{v_{I I}}{1+\left(M_{\mathrm{t}} / M_{\mathrm{p}}\right)^{3}}$.

$M_{\mathrm{t}}$ is the transition mass between Type I and Type II migration (Ward 1997) and is set to

$M_{\mathrm{t}}=0.4 \alpha^{2 / 3}\left(H / R_{\mathrm{p}}\right)^{-1 / 3}$.

The migration time-scale is therefore

$\tau_{I, I I}=\frac{R_{\mathrm{p}}}{\left|v_{I, I I}\right|}$.

We calculate the migration time-scales from the above equations assuming a planet at $R_{\mathrm{p}}=5.2$ au in a disc with $\alpha_{\Sigma}=0.5$, $\Sigma_{\mathrm{p}}=75 \mathrm{~g} \mathrm{~cm}^{-2}$, and $H_{\mathrm{p}} / R_{\mathrm{p}}=0.05$. In Fig. 8, we plot three cases that correspond to different disc viscosity $(\alpha=0.1,0.01,0.002)$. We note that these calculations are for planets in low-mass discs at specific regimes: Tanaka et al. (2002) study 3D discs including both Lindbland and corotational resonances assuming an isothermal disc, whereas Ward (1997) studies a 2D disc ignoring corotational resonances. These calculations are not applicable for the cases we examine in this paper. Therefore, these analytic solutions are plotted in the graph in Fig. 8 merely for reference, so as to put our results in context with the classic picture of Type I and Type II migration.

The migration time-scales in Fig. 8 are plotted for five different times in the simulation: $0.7,1.5,2.5,5$, and $18 \mathrm{kyr}$. As the mass of the protoplanet increases with time, the same graph may be used to track the evolution of the migration time-scale with time. In all runs, the protoplanet initially migrates inwards on time-scales similar to the Type I migration time-scale of a $1-M_{\mathrm{J}}$ planet, i.e. $\sim(1-2) \times 10^{4} \mathrm{yr}$. As the protoplanet moves towards the host star and grows in mass, the migration time-scale becomes shorter (down to $\sim 3 \times 10^{3} \mathrm{yr}$ ), but once the gap opens up the migration slows down and eventually the protoplanet starts moving outwards in all runs apart from the case when its radiative feedback is taken into account. In this case, inward 


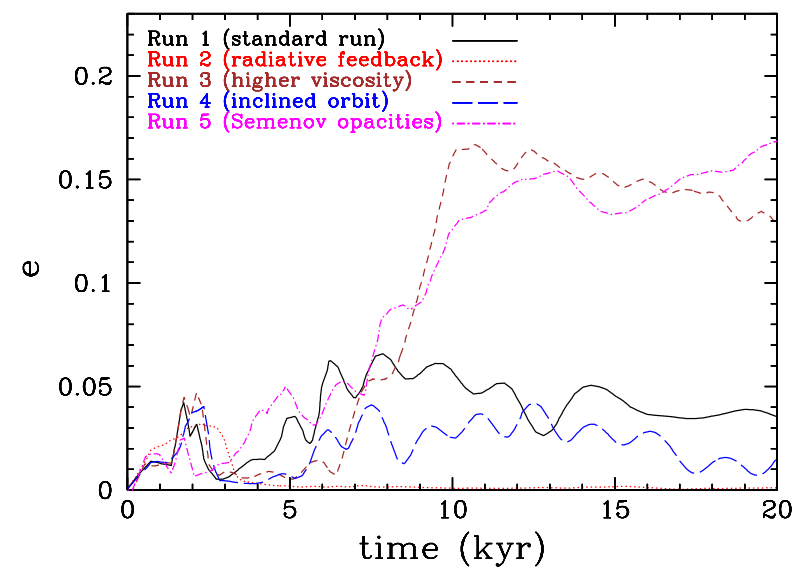

Figure 9. The protoplanet eccentricity increases with time in all runs apart from the case with the protoplanet feedback. The eccentricity growth is due to interactions with the gravitationally unstable gap edges. When the protoplanet radiative feedback is taken into account the gap edges are stable and the orbit of the protoplanet ends up almost circular.

migration continues albeit at a much longer time-scale, similar to the Type II migration case $\left(\sim 2 \times 10^{5} \mathrm{yr}\right)$.

\subsection{Eccentricity}

The eccentricity of the protoplanet grows as the protoplanet interacts with the gravitationally unstable disc (see Fig. 9). The growth pattern is rather stochastic with the eccentricity growing up to $\sim 0.15$. Even if the eccentricity after this initial growth period dampens with time due to secular interactions with the disc, these initial interactions can provide the seed for subsequent eccentricity growth (Goldreich \& Sari 2003; Duffell \& Dong 2015). Therefore, discplanet interactions, while the disc is still relatively massive, may help explain the observed high eccentricities of giant planets in systems that are thought to contain only one planet (Wright et al. 2011; Dunhill \& Stamatellos 2018). In the case when the radiative feedback of the protoplanet is taken into account (Run 2), the eccentricity initially grows during the gap opening phase but then it is dampened quickly, as there is no strong stochastic driving, so that the protoplanet ends up in a nearly circular orbit. Therefore, interactions within a gravitationally unstable disc is necessary for eccentricity growth to occur.

\subsection{Protoplanet on an inclined orbit}

The protoplanet in the run in which its orbit is inclined (Run 4, blue lines) shows mass growth that resembles closely the standard run (see Fig. 3). This is because the protoplanet's orbit inclination gets smaller quickly as the protoplanet crosses through the disc mid-plane. Within $\sim 2$ kyr ( $\sim 5$ orbits) the protoplanet's orbit has been aligned with the disc mid-plane (inclination has become zero; see Fig. 10). Therefore, the long-term evolution of a protoplanet is not significantly different if the protoplanet initially forms on an inclined orbit in a relatively massive protostellar disc.

\subsection{Gap opening}

The surface density, the temperature profile, and the Toomre parameter $Q$ for the region around the protoplanet for all five runs are shown in Fig. 11 (on the protoplanet's corotational frame). The snapshots correspond to $t=8 \mathrm{kyr}$, i.e. after the gap has been opened

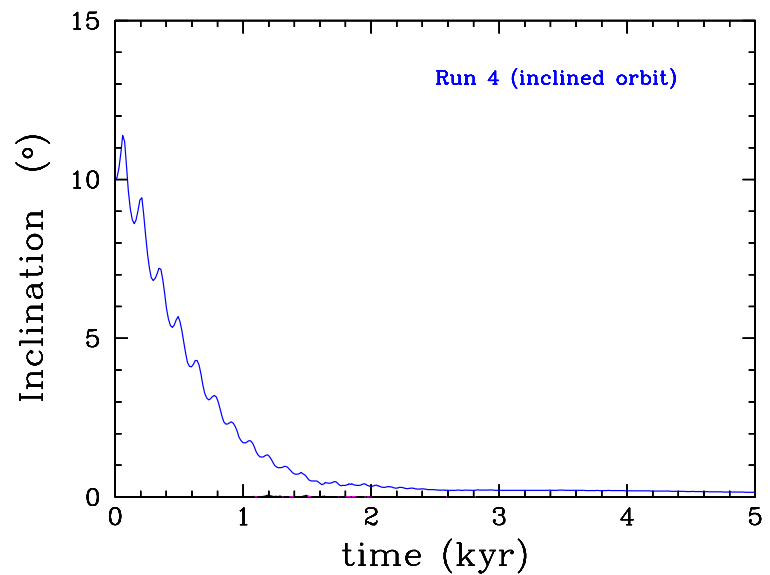

Figure 10. The evolution of the protoplanet's orbit inclination with respect to the disc mid-plane, for Run 4. The protoplanet's orbit quickly aligns with the disc mid-plane.

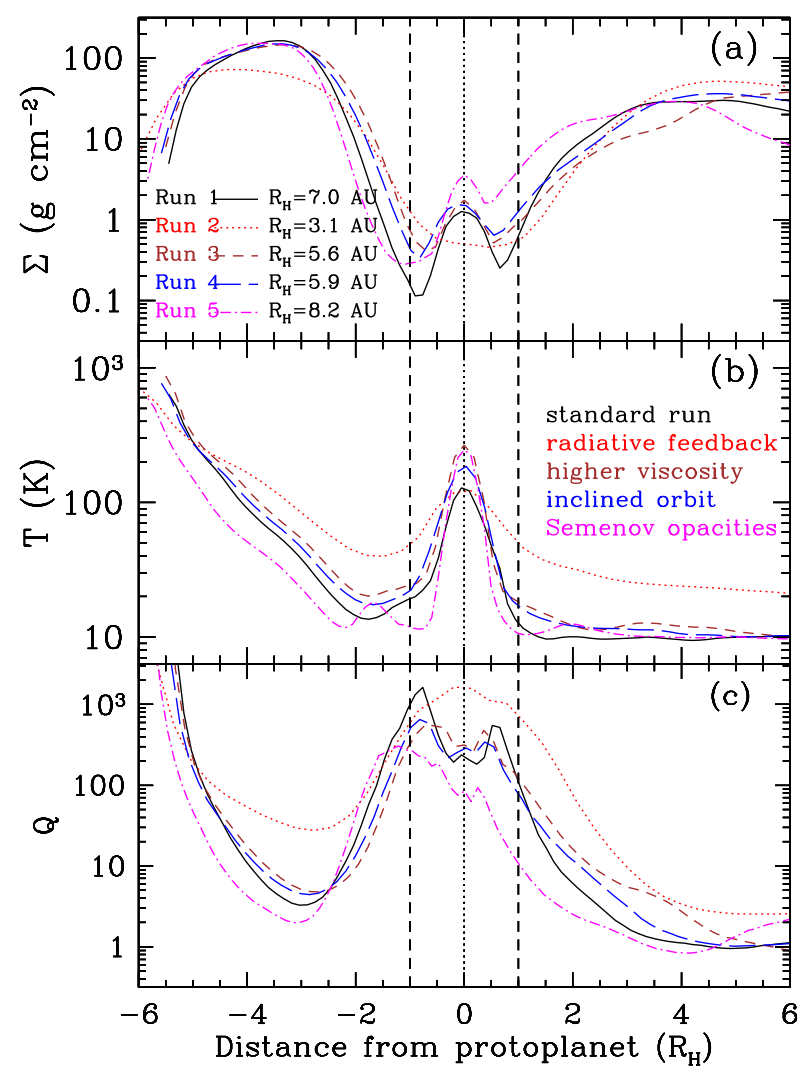

Figure 11. The surface density (a), the temperature at the disc mid-plane (b), and the Toomre $Q$ parameter (c), in the protoplanets corotational frame, as a function of the distance from the protoplanet, in units of its Hill radius, for the simulations listed in Table 1. The protoplanet is at $r=0$, whereas negative values correspond to the direction towards the central star. All simulation snapshots are at time $t=8 \mathrm{kyr}$, i.e. after a gap has been established in the discs. The values of the Hill radius of the protoplanet for each run are also shown on the top graph.

up in the disc. The gap size is a few Hill radii and rather similar for all runs (note though that the size of the Hill radius is different in each run, as the protoplanet is on different orbits in different runs). The increase of surface density towards the protoplanet within the 


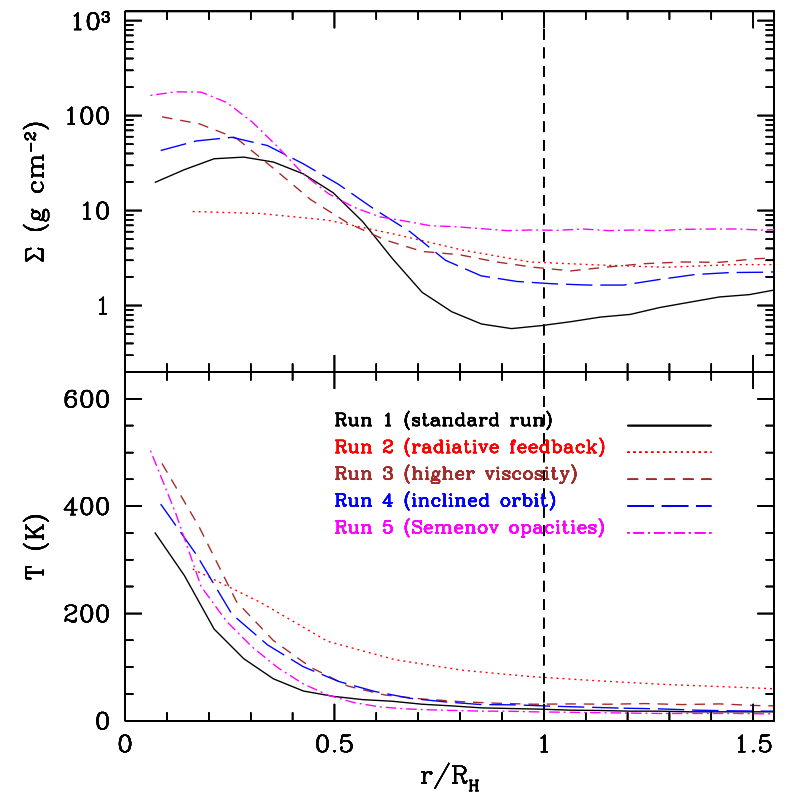

Figure 12. The properties of the circumplanetary disc in the simulations listed in Table 1 at $t=8 \mathrm{kyr}$. Azimuthally averaged surface density $\Sigma$ (top) and temperature $T$ (bottom) as a function of the distance from the protoplanet (in units of its Hill radius).

Hill radius corresponds to the circumplanetary disc (see section below).

The surface density at the boundaries of the Hill radius is asymmetric (Fig. 11a). In Run 2 (which includes the protoplanet's radiative feedback), the surface density at the inner boundary of the protoplanet's Hill radius (e.g. at $-1.5 R_{\mathrm{H}}$ ) is higher than the surface density at outer boundary of the Hill radius (e.g. at $+1.5 R_{\mathrm{H}}$ ), whereas in all other runs the outer boundary surface density is higher. This is consistent with the point made in the previous subsection, i.e. that a large fraction of gas is accreted on to the protoplanet from outside its orbit when the gap edges are gravitationally unstable. The asymmetry is more pronounced for Run 5 (higher opacity run) which also exhibits shorter outward migration timescales (see Table A1 and Fig. 8). The disc temperature (Fig. 11b) at the run with the protoplanet feedback is higher than in the other runs, which results in stabilizing the disc edges as the $Q$ parameter is larger than $\sim 3$ (Fig. 11c). The temperature of the disc around the protoplanet is lower for the higher opacity run (Run 5) which helps opening up the gap fast and results in higher gas accretion on to the protoplanet.

\subsection{Circumplanetary discs}

The properties of the circumplanetary discs in all five runs are shown in Fig. 12 and the time evolution of the Hill radius and the mass within it for the protoplanet in each run are shown in Figs 13 and 14. The typical circumplanetary disc mass is about $0.1 M_{\mathrm{J}}$, which means that the disc is resolved by $\sim 1000$ SPH particles, i.e. approximately seven smoothing lengths (assuming a nearly 2D disc). The sink radius of the protoplanet is set to 0.1 au so it is always smaller than the Hill radii of the protoplanets at all times, in all runs (see Fig. 13). The circumplanetary discs are therefore just resolved and the presented properties near the planet are probably resolution dependent. We expect that the surface density and the temperature near the planet are underestimated. In Run 2 (with protoplanet feedback), the circumplanetary disc is smaller (mass

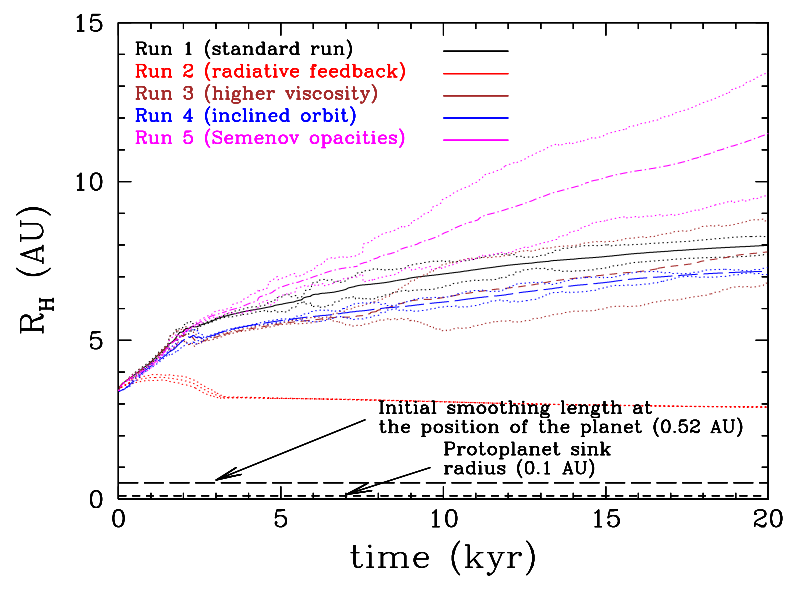

Figure 13. The evolution of the Hill radius of the protoplanet. The solid lines correspond the values calculated using the semimajor axes of the protoplanet, whereas the dotted lines correspond to the periastron and apoastron of the protoplanet's orbit. The actual Hill radius of each protoplanet varies between these two extremes.

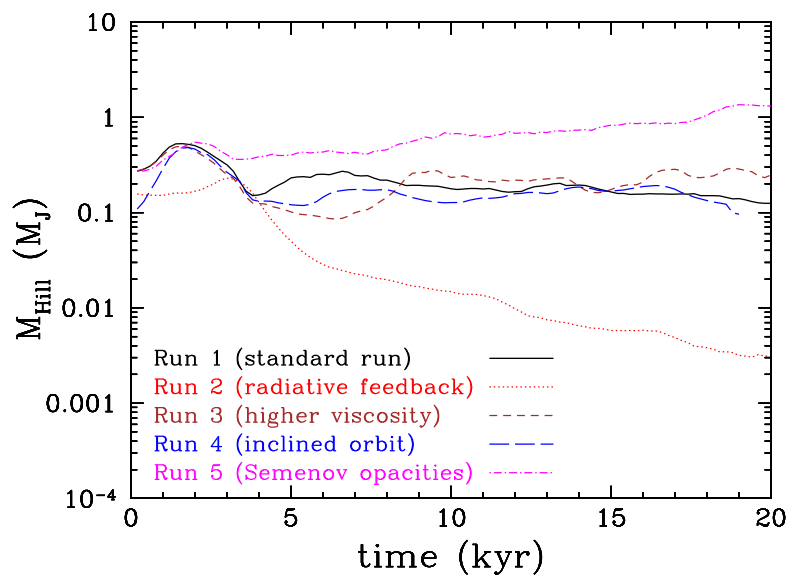

Figure 14. The mass within the protoplanet's Hill radius as a function of time for the simulations listed in Table 1.

$0.01 M_{\mathrm{J}}$; see Fig. 14) as the protoplanet is closer to the parent star; therefore, the circumplanetary disc is just resolved in this case (only by $\sim 2$ smoothing lengths).

Despite the above drawback, by comparing the derived properties we see that in Run 2, with the protoplanet radiative feedback, the circumplanetary disc is hotter than in the other runs, as expected. Radiative feedback from the protoplanet together with the gas thermodynamics (Gressel et al. 2013) is important in determining the properties of circumplanetary discs. Further studies with higher resolution are needed for more secure results. Additionally, the presence of magnetic field may also play a significant role (Gressel et al. 2013; Fujii et al. 2014) but we do not examine this case here.

We note that we do not find temperatures in the circumplanetary discs higher than a few hundred Kelvin in contrast to the simulations of Szulágyi (2017), in which they find that temperatures close to the protoplanet rise up to a few thousand Kelvin. However, their simulations are able to resolve the region down to $10^{-3} \times R_{\mathrm{Hill}}$, which can indeed become very hot. We note though that the Szulágyi (2017) simulations do not include the effect of molecular hydrogen dissociation at $\sim 2000 \mathrm{~K}$ (nor the ionization of hydrogen and the first and second ionization of helium), so she may overestimate the 

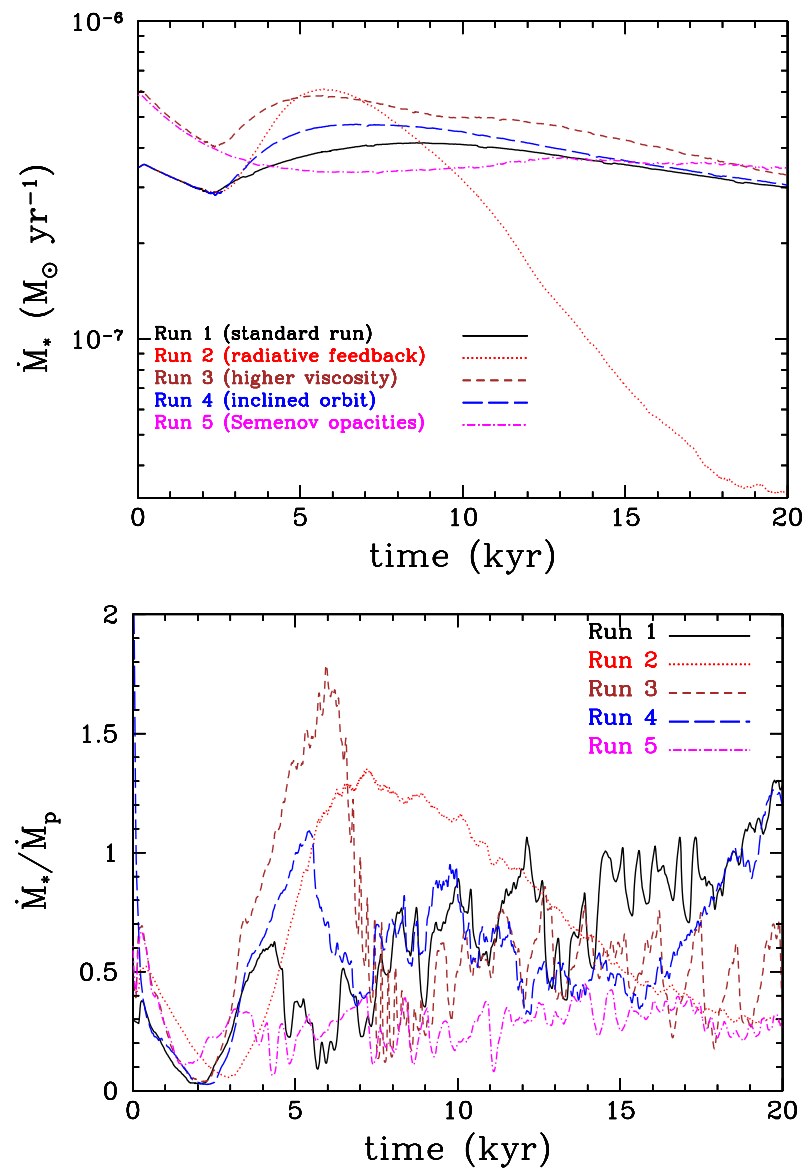

Figure 15. The accretion rate on to the central star (top) and the ratio of the accretion rate on to the star to the accretion rate on to the protoplanet (bottom). The accretion rate is higher for higher viscosity (Run 3) or for higher opacity (Run 5).

temperatures in the inner region. When we compare the temperatures farther out in the circumplanetary disc (e.g. at $0.5 \times R_{\mathrm{Hill}}$ ), we still see that the temperatures we find here are lower than her temperatures.

\subsection{Accretion on to the central star}

The presence of a protoplanet in the disc regulates the accretion rate on to the star (see Fig. 15). Interestingly, the accretion rate on to the protoplanet during the initial stages of its evolution that we model here, is comparable to the accretion rate on to the star itself. During the gap opening phase it can be even ten times higher. This is consistent with previous studies of planets embedded in lower mass discs (Nelson et al. 2000; Lubow \& D'Angelo 2006; Owen 2014). During this phase the accretion rate on to the star decreases but as the protoplanet moves closer to the star and once the gap opens up, the accretion rate on to the star increases by a factor of a few, as the protoplanet acts to drive accretion on to the star; the closer the protoplanet orbit to the star and the higher its mass, the higher the accretion rate on to the star becomes. At this stage, and for a few thousand years, the accretion rate on to the star may become higher than the accretion rate on to the protoplanet. In Run 5 (with the Semenov opacities), the protoplanet stays sufficiently away from the star so that the accretion rate on to the star is not affected.

In Run 2 (with protoplanet feedback), the protoplanet migrates closer to the star than in the other runs, and the accretion rate on to the star increases significantly until a cavity forms around the star; thereafter, the accretion rate drops considerably as the presence of the protoplanet starves the star from gas. At the same time, the protoplanet experiences similar gas starvation as it resides within the same cavity. This stage can be thought as similar to the transition disc phase (see review by Espaillat et al. 2014). However, the accretion rate on to the protoplanet is still higher than the accretion on to the star by a factor of 2 . In this case the star-protoplanet system behaves as a low mass ratio binary system with a circumbinary disc, in which the secondary component (i.e. the protoplanet) accretes more than the primary component (i.e. the star; Artymowicz \& Lubow 1994; Artymowicz \& Lubow 1996). In such systems, secondaries increase in mass faster than primaries, resulting in an almost equal-mass binaries (e.g. Satsuka et al. 2017). However, in the case of a planetary-mass companion, its mass cannot become comparable to that of the star; even if an unlikely high accretion rate of $\sim 10^{-4} M_{\mathrm{J}} \mathrm{yr}^{-1}$ is maintained for $\sim 10^{5} \mathrm{yr}$, then the protoplanet's mass will increase only by $10 M_{\mathrm{J}}$.

\subsection{The role of the accretion rate on to the protoplanet}

The actual accretion rate on to the protoplanet is important as it regulates the strength of its radiative feedback, which in turn determines whether the edges of the gap opened by the protoplanet are gravitationally unstable (so that the protoplanet migrates outwards), or gravitationally stable (so that protoplanet migrates inwards). The luminosity of the protoplanet, $L_{\mathrm{p}}$, is proportional to the accretion rate, $\dot{M}_{\mathrm{p}}$, on to the protoplanet (see equation 7), therefore the temperature due to the presence the protoplanet (see equation 6) scales as $T_{\mathrm{A}}^{\text {planet }}(r) \propto \dot{M}_{\mathrm{p}}^{1 / 4}$. The Toomre parameter, $Q$, that determines whether the gap edges are gravitationally unstable is $Q(r) \equiv$ $\kappa c_{\mathrm{s}}(r) / \pi G \Sigma(r)$, where $\kappa$ is the epicyclic frequency, and $c_{\mathrm{s}}$ is the sound speed (we assume that the distance, $r$, is measured from the protoplanet). Hence, $Q \propto\left[T_{\mathrm{A}}^{\mathrm{planet}}(r)\right]^{1 / 2}$ or equivalently

$Q \propto \dot{M}_{\mathrm{p}}^{1 / 8}$.

Therefore, there is only a weak dependence of the Toomre parameter on the accretion rate on to the protoplanet. If we assume that the actual accretion rate is 10 times lower than the one we estimate in the simulations we present here, then the $Q$ value is lower only by a factor of $\sim 1.3$ (assuming that the other parameters remain the same). Then, the $Q$ value in the outer edge of the gap in the run with the protoplanet radiative feedback (see Fig. 11, red line) will drop from 3 to 2.3 , i.e. the gap edge will still be gravitationally stable $(Q>1.5)$. We conclude the value of the accretion rate on to the protoplanet is not critical, at least qualitatively, regarding the effect of radiative feedback on the migration of the protoplanet.

\section{COMPARISON WITH $\beta$-COOLING STUDIES}

We compare the results of the simulations presented here with previous studies that employ the $\beta$-cooling approximation, in which the cooling time in the disc is proportional to the local orbital period. In this case, the specific internal energy of each SPH particle is set to

$u=\frac{k_{B} T(R)}{\mu m_{\mathrm{H}}(\gamma-1)}$,

where $\mu=2.45$ is the mean molecular weight, and $\gamma=7 / 5$ is the adiabatic exponent. The cooling rate of each particle is set to

$\left.\frac{\mathrm{d} u}{\mathrm{~d} t}\right|_{\text {cool }}=-\frac{u}{t_{\text {cool }}}$ 

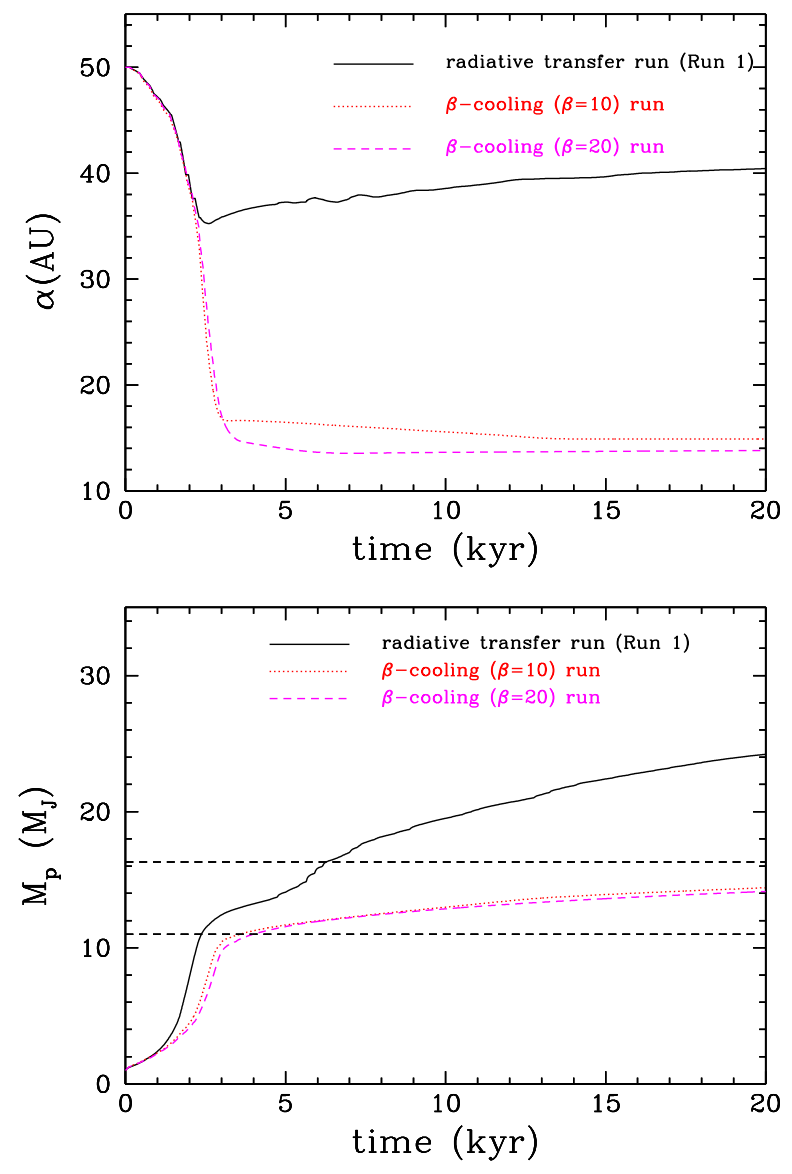

Figure 16. The evolution of a protoplanet in a disc in a run with radiative transfer (Run 1) and in two runs using the $\beta$-cooling approximation. Semimajor axis (top) and protoplanet mass (bottom) are plotted against time. In the $\beta$-cooling runs, the cooling times are long, the protoplanet does not open up a gap and migrates fast in the inner disc region close to the star.

where

$t_{\mathrm{cool}}=\beta \Omega_{K}^{-1}(R)$,

$R$ is the distance on the disc mid-plane, and

$\Omega_{K}(R)=\left(\frac{G M_{\star}}{R^{3}}\right)^{1 / 2}$.

We perform simulations with $\beta=10$ and $\beta=20$, i.e. relatively long cooling times. These are similar to the ones used before in these type of studies: Baruteau et al. (2011) use $\beta=15,20,30$, whereas Malik et al. (2015) use $\beta=30$. Otherwise, the parameters of the simulations are the same as in Run 1 . We note that considering these long cooling times the discs are gravitationally stable and they are not expected to show any spiral structure in the absence of the protoplanet. We compare the simulation we performed with the results in Run 1 in which radiative transfer is treated self-consistently with the method of (Stamatellos et al. 2007, see Section 3.1).

We see (Fig. 16) that in the runs that use the $\beta$-cooling approximation the protoplanet is not able to open up a gap and the migration is fast, as previous studies have found, and stops only once the protoplanet has reached the inner cavity around the star. During migration, the accretion on to the protoplanet is much lower than in the runs in which the radiative transfer is treated in more detail. The reason for this is that in the $\beta$-cooling runs (that as in previous studies use rather large $\beta$ values; $15,20,30)$, the actual cooling is

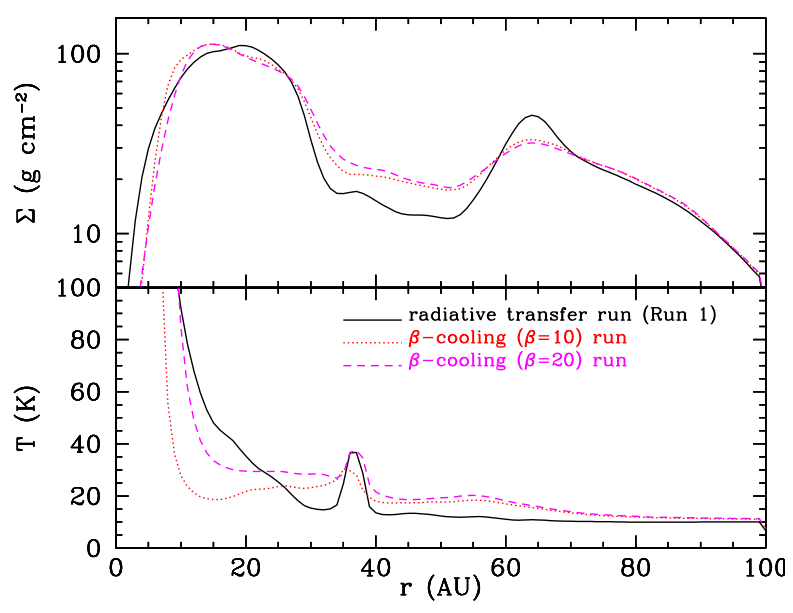

Figure 17. Azimuthally averaged disc surface density (top) and temperature (bottom), for the three runs in Fig. 15 at $t=2 \mathrm{kyr}$ (i.e. during the initial gap opening phase). The protostellar disc is hotter in the runs that use the $\beta$-cooling approximation, so that gap opening is more difficult than in the run with a more detailed radiative transfer (Run 1). The outer regions of the protoplanetary disc (peak at $\sim 37$ au in the temperature plot) are also hotter due to inefficient cooling, limiting gas accretion on to the protoplanet.

too slow compared with the cooling provided by the more detailed method, so that the protostellar disc is hotter (see Fig. 17, bottom) and therefore the opening of a deep gap is not possible (Crida et al. 2006). Additionally, the cooling in the outer circumplanetary disc is inefficient, resulting in slower accretion of gas on to the protoplanet, limiting its mass growth. We have already discussed in detail the effect of cooling on the mass growth of the protoplanet and on gap opening in Section 4.1.

Therefore, the detailed treatment of radiation transfer in the disc and the vicinity of the protoplanet, i.e. how the circumstellar and circumplanetary discs heat and cool, is important for determining the migration and mass growth of a protoplanet evolving in a young, massive disc.

\section{THE EFFECT OF THE PROTOPLANET'S ORBITAL RADIUS (WITHOUT RADIATIVE FEEDBACK FROM PROTOPLANET/STAR}

We now examine the evolution of Jupiter-mass protoplanets that are embedded at different orbital radii within protostellar discs (see Fig. 18). The details of the five runs (hereafter referred to as sRuns) are shown in Table 2. A protoplanet is initially placed at 5, 10, 20, 50 , and 80 au from the central star at a circular orbit. The opacities used for these runs are the ones by Semenov et al. (2003). For these runs, we do not include radiative feedback from the star nor the protoplanet. The migration time-scales for each run and the associated migration velocities are shown in Tables A3 and A4.

The semimajor axis evolution of the protoplanets is shown in Fig. 19. When the protoplanet is initially placed in the outer disc region (which is characterized by a low Toomre- $Q$ parameter), it migrates inwards on a Type I migration time-scale $\left(\sim 10^{4} \mathrm{yr}\right)$, but once a gap opens up the inward migration stops and it changes into outward migration (see Fig. 20). On the other hand, when the protoplanet is placed in the inner disc region $(\lesssim 20 \mathrm{au})$ it migrates inwards initially on a Type I migration time-scale $\left(\sim 10^{4} \mathrm{yr}\right)$ and once the gap is opened up the migration continues to be inwards; however, it slows down and occurs on a time-scale typical of Type II migration $\left(\sim 10^{5} \mathrm{yr}\right.$; see Fig. 20$)$. As pointed out in the previous 


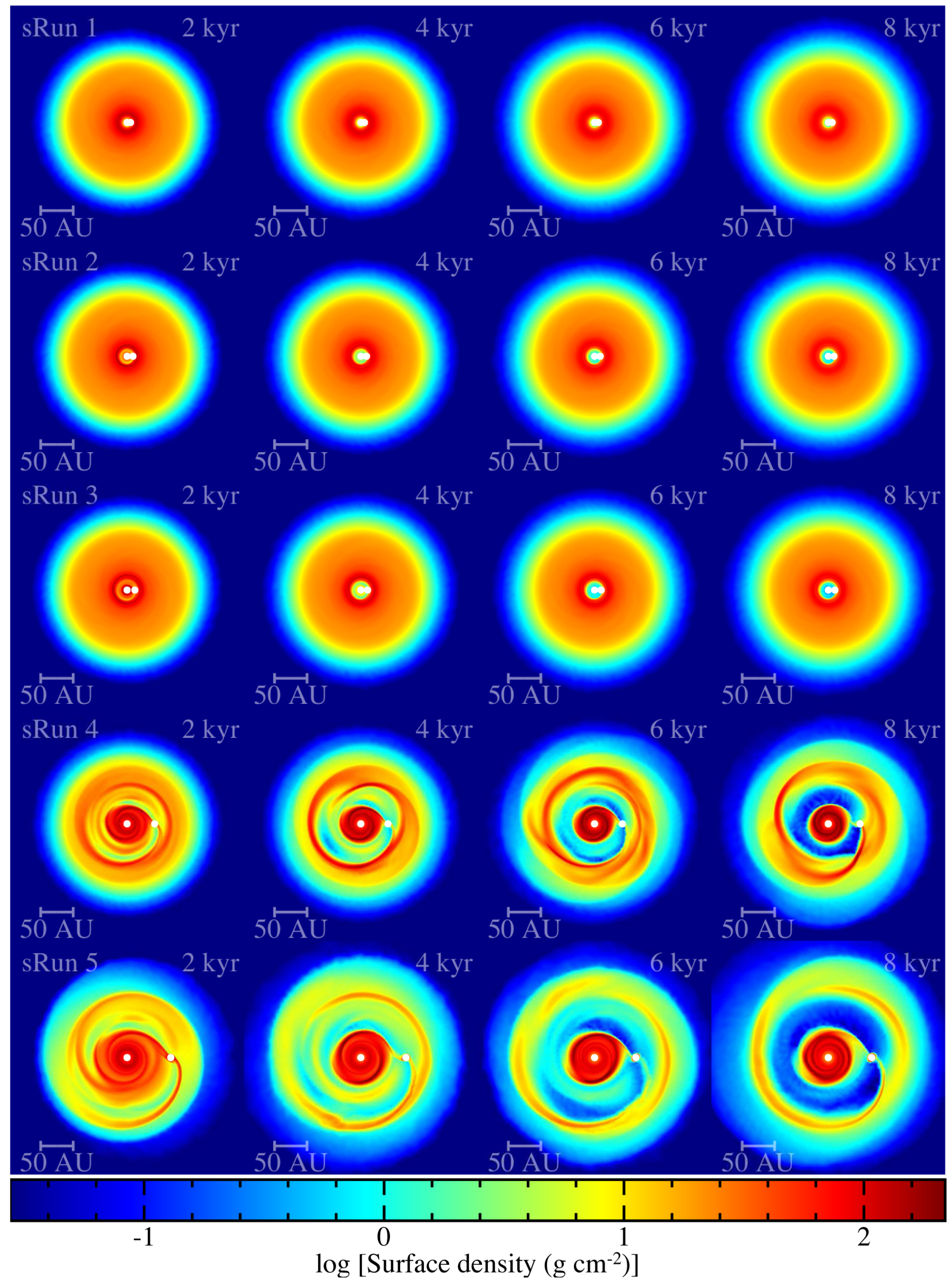

Figure 18. The evolution of a protoplanet in a $0.1-\mathrm{M}_{\odot}$ protostellar disc. The protoplanet is placed at different initial orbital radii within the disc: 5 , 10, 20, 40 , and 80 au (top to bottom row; sRun1, .., sRun5, respectively). There is a difference in the migration pattern depending on the position of the protoplanet in the disc. 
Table 2. Evolution of a protoplanet at different orbital radii within a disc (without radiative feedback): simulation parameters (same as in Table 1).

\begin{tabular}{lccccl}
\hline Run id & $\begin{array}{c}M_{p, i} \\
\left(M_{\mathrm{J}}\right)\end{array}$ & $\begin{array}{c}\alpha_{i, p} \\
(\mathrm{au})\end{array}$ & $\begin{array}{c}M_{p, f} \\
\left(M_{\mathrm{J}}\right)\end{array}$ & $\begin{array}{c}\alpha_{f, p} \\
(\mathrm{au})\end{array}$ & $e_{f}$ \\
\hline sRun1 & 1 & 5 & 6.8 & 6.7 & 0.0013 \\
sRun2 & 1 & 10 & 9.4 & 8.8 & 0.007 \\
sRun3 & 1 & 20 & 9.1 & 9.9 & 0.0002 \\
sRun4 & 1 & 50 & 31 & 53 & 0.17 \\
sRun5 & 1 & 80 & 26 & 72 & 0.10 \\
\hline
\end{tabular}

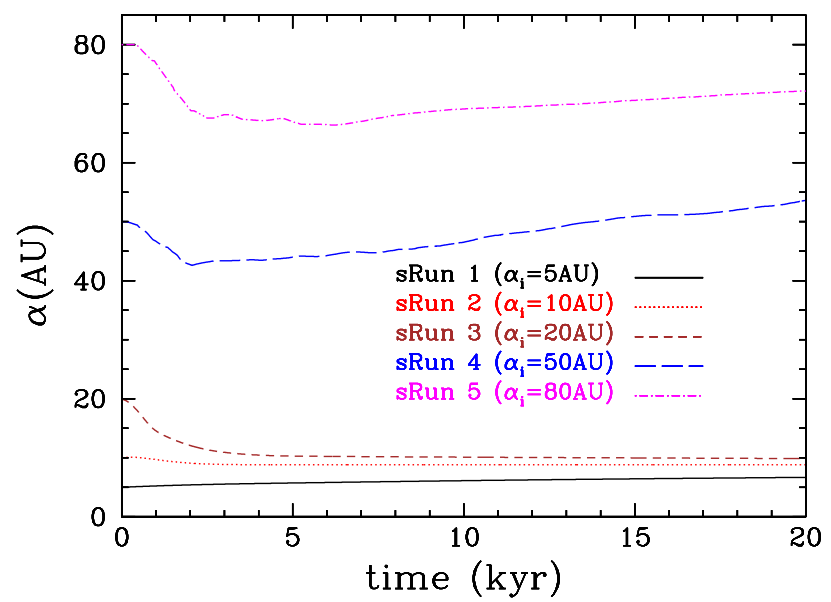

Figure 19. The semimajor axis evolution of a $1-M_{\mathrm{J}}$ protoplanet in a $0.1-$ $M_{\odot}$ disc. When the protoplanet is placed in the outer disc region, it migrates inwards on a Type I migration time-scale. However, when the gap opens up, inward migration stops and it reverses into an outward migration. On the other hand, when the protoplanet is placed in the inner disc region $(\lesssim 20 \mathrm{au})$, it migrates inwards initially on a Type I migration time-scale and once the gap is opened up the migration slows down and occurs on a Type II time-scale.

section, a necessary requirement for outward migration is interactions with a gravitationally unstable outer gap edge. In the inner disc region close to the central star the disc is hotter and therefore stable, so that outward migration does not happen. The only exception is the run in which the protoplanet initially placed at $5 \mathrm{au}$; in this run, the protoplanet migrates outwards (but only slightly) throughout the simulated time because it resides near the outer edge of a quickly formed inner cavity around the central star. It accretes less gas than in the other runs; mainly it accretes high angular momentum gas from the outer edge of the cavity, as indicated by the lack of strong inner wake.

We conclude that the survival of the protoplanet on a relatively wide orbit (assuming that it has formed on this wide orbit) is secured when the disc is massive and cool enough for gravitationally unstable gap edges to develop. If the protoplanet forms in the outer disc region then its initial inward migration will turn into an outward migration once it opens up a gap in the disc. If the protoplanet forms in the inner disc region, its inward migration will slow down considerably (migration time-scale of $\gtrsim 10^{5} \mathrm{yr}$; see Table A3) so it can stay on a wide orbit once the disc has dissipated (assuming that the disc dissipates fast enough).

The mass growth of the protoplanet also depends on its location in the disc (see Fig. 21). Protoplanets placed in the outer disc region accrete gas while they open up a gap and they continue to accrete gas at a high rate as they migrate outwards in the disc (Fig. 22, top).

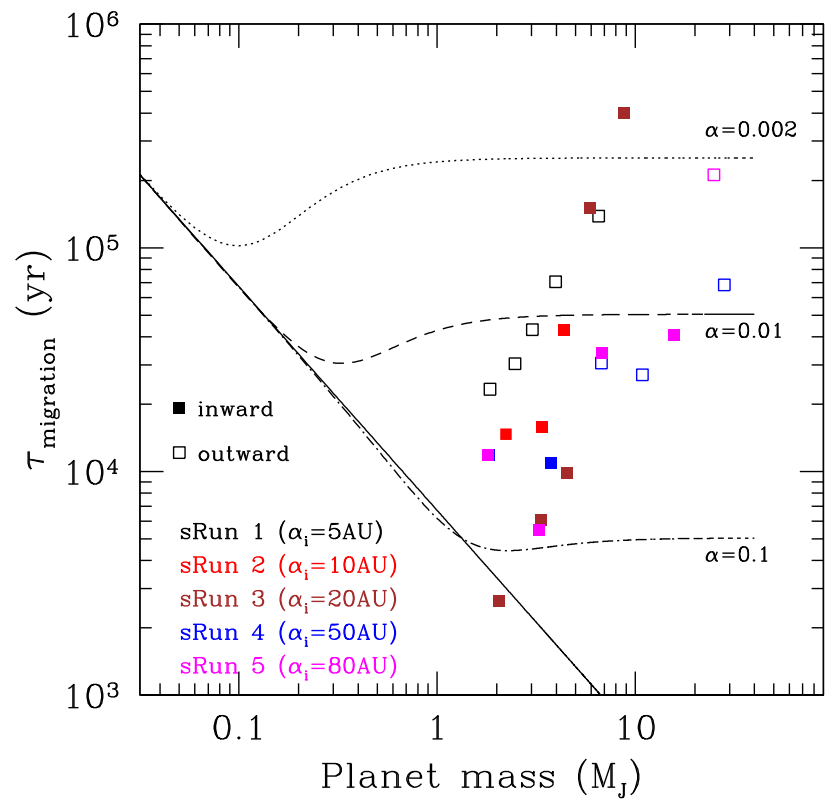

Figure 20. The migration time-scales of protoplanets placed at different orbital radii within a protostellar disc. Protoplanets initially migrate inwards. If the protoplanet is in the unstable outer disc region the migration stops and changes outwards once a gap is opened up. Protoplanets that are in the inner disc region ( $\lesssim 20 \mathrm{au}$ ) continue to migrate inwards. Lines correspond to analytical calculations as in Fig. 8. Filled boxes correspond to inward migration and empty boxes to outward migration.

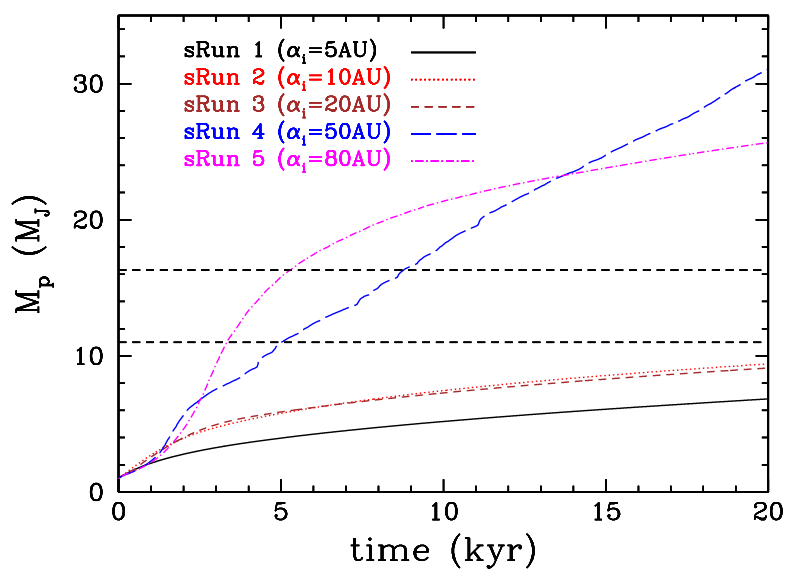

Figure 21. Mass growth of a $1-M_{\mathrm{J}}$ protoplanet placed at different radii within a protostellar disc. Protoplanets that form in the outer disc regions tend to increase in mass considerably, becoming brown dwarfs. Protoplanets in the inner disc region increase in mass but not significantly enough to become brown dwarfs. The horizontal dashed lines correspond to the deuteriumburning mass-limit.

They increase in mass considerably becoming brown dwarfs by the end of the simulated $20 \mathrm{kyr}$, with a mass of $\sim 25-30 M_{\mathrm{J}}$. Their corresponding accretion luminosities are $\gtrsim 0.1 \mathrm{~L}_{\odot}$ (see also Inutsuka et al. 2010, Fig. 22) and therefore they may be readily observable if observed while they are still young. On the other hand, protoplanets that form in the inner disc region quickly find themselves within a gas-poor cavity formed around the central star and accretion on to them happens at a much lower rate than on protoplanets in the outer disc region (Fig. 22). Their mass growth is slower and their final mass at the end of the simulation is below the deuteriumburning limit $\left(8-10 M_{\mathrm{J}}\right)$. However, their mass continues to increase 

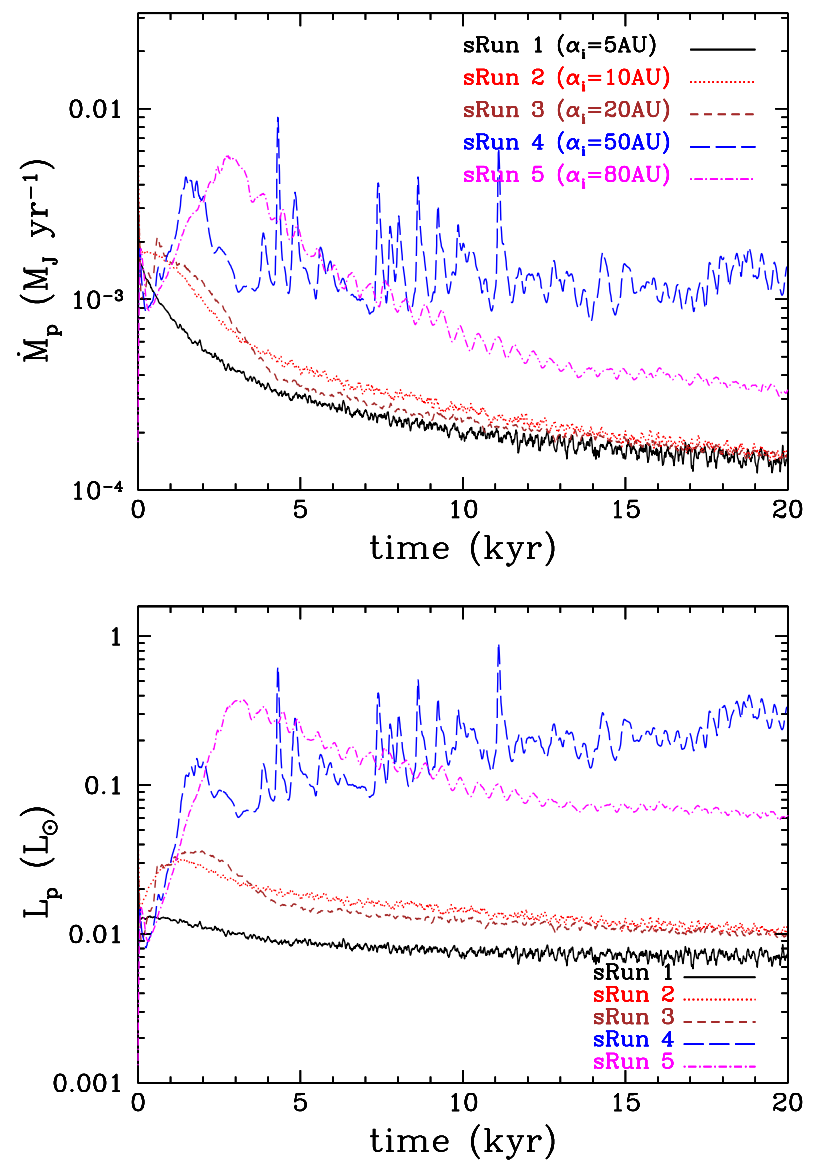

Figure 22. Accretion rate (top) on to $1-M_{\mathrm{J}}$ protoplanet placed at different radii within a protostellar disc, and its corresponding accretion luminosity (bottom). Protoplanets in the outer disc region continue to accrete gas vigorously even after they open up a gap in the disc. Protoplanets in the inner disc region accrete significantly less as they reside within a cavity formed around the central star (see Fig. 18 - top three rows). (Note that in this set of runs the protoplanet's luminosity is not fed back into the disc.)

so they may also end up as brown dwarfs if the disc dissipates slowly. If the longer term disc dissipation happens on a shorter timescale than the viscous one in other ways, e.g. by photoevaporation (Alexander, Clarke \& Pringle 2006; Owen, Ercolano, Clarke \& Alexander 2010) or by disc winds (Suzuki \& Inutsuka 2009, 2014; Suzuki, Muto \& Inutsuka 2010; Gressel et al. 2015; Bai 2016, see review by Alexander et al. 2014), then the mass of the protoplanet will remain below the deuterium-burning limit (see discussion by Kratter et al. 2010).

The eccentricity of the protoplanet in the different runs is shown in Fig. 23. Protoplanets in the outer disc region interact with the gravitationally unstable gap edges resulting in eccentricity growth $(e \gtrsim 0.1)$. Protoplanets in the inner disc region interact in a gravitationally stable disc and their orbits remain nearly circular. Therefore, for a protoplanet to have a significantly eccentric orbit it needs to have formed at a sufficiently large distance from the central star. Protoplanets formed by gravitationally instability naturally form at such large radii when the disc can be both gravitationally unstable and cool fast enough (Boley 2009; Stamatellos \& Whitworth 2009a). Giant planets are difficult to form by core accretion at such large radii. Therefore, eccentric giant planets on wide orbits in single-planet systems may have formed due to gravitational instabilities.

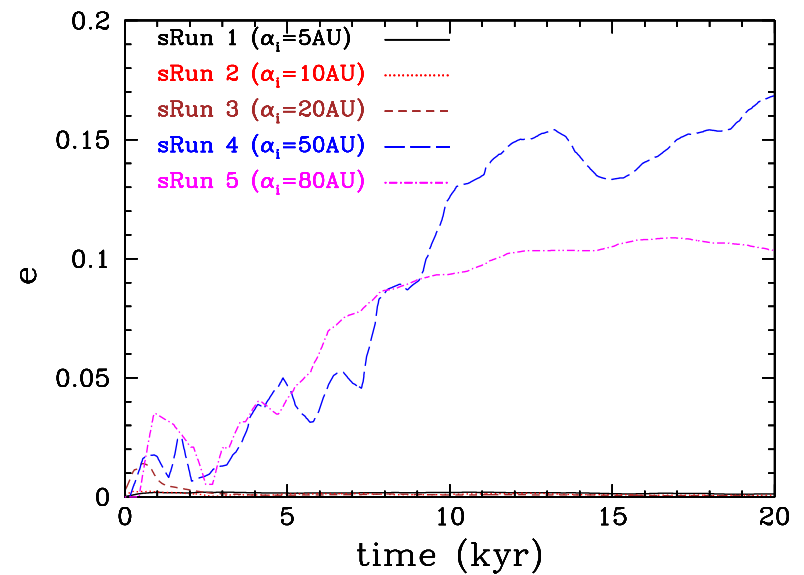

Figure 23. The eccentricity of a $1-M_{\mathrm{J}}$ protoplanet placed at different radii within a protostellar disc. If the protoplanet is in the outer disc region, interactions with the gravitationally unstable gap edges result in eccentricity growth. If the protoplanet is in the inner disc region interactions with a stable disc result in a circular orbit.
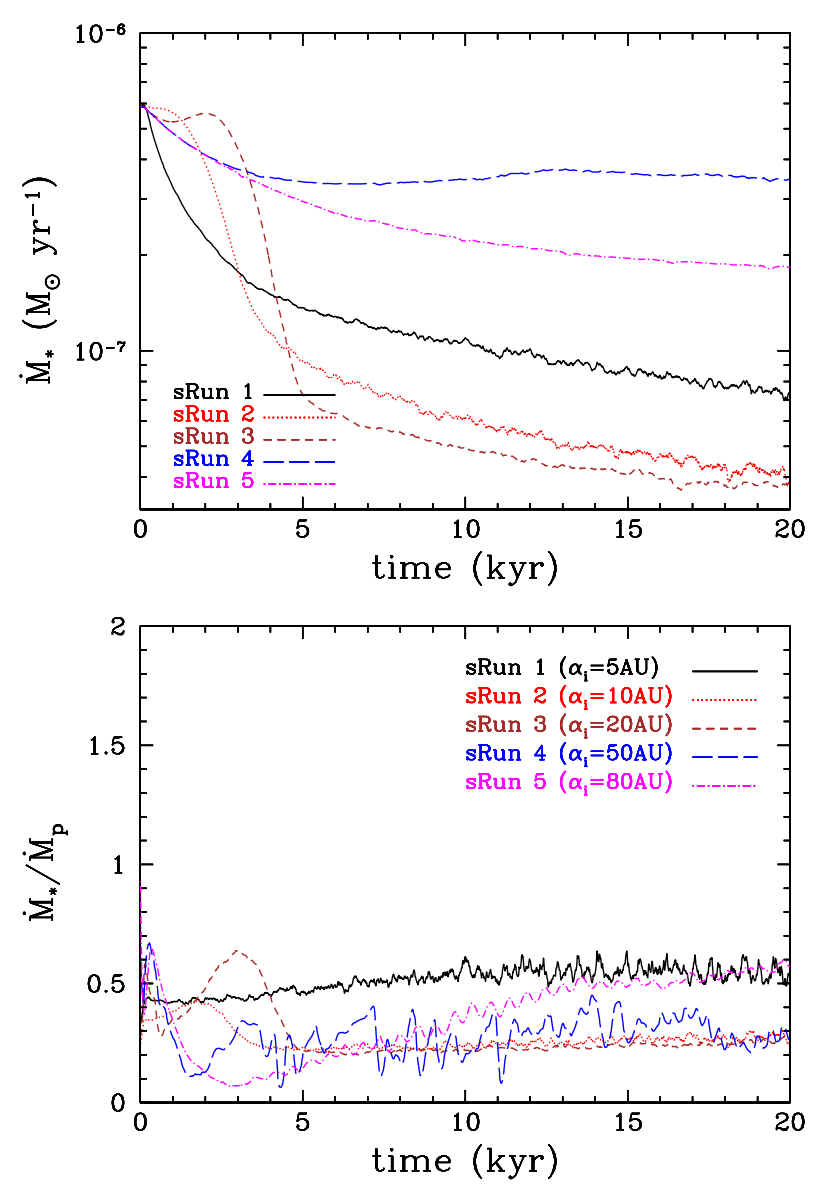

Figure 24. The accretion rate on to the central star (top) and the ratio of the accretion rate on to the star to the accretion rate on to the protoplanet (bottom). The accretion rate on to the star is lower when the protoplanet is in the inner disc region. Generally, the star accretion rate is only half (or less than) the accretion rate on to the protoplanet.

The accretion rate on to the central star also depends on the orbital radius of the protoplanet (Fig. 24). A protoplanet in the inner disc region quickly (within a few kyr) forms a cavity around the central star. During this period the accretion rate on to the star is high, as 
Table 3. Evolution of a protoplanet at different orbital radii within a disc (with radiative feedback from the star and the protoplanet): simulation parameters (same as in Table 1).

\begin{tabular}{lccccc}
\hline Run id & $\begin{array}{c}M_{p, i} \\
\left(M_{\mathrm{J}}\right)\end{array}$ & $\begin{array}{c}\alpha_{p, i} \\
(\mathrm{au})\end{array}$ & $\begin{array}{c}M_{p, f} \\
\left(M_{\mathrm{J}}\right)\end{array}$ & $\begin{array}{c}\alpha_{p, f} \\
(\mathrm{au})\end{array}$ & $e_{f}$ \\
\hline rRun1 & 1 & 5 & 8.0 & 6.6 & 0.0020 \\
rRun2 & 1 & 10 & 11.4 & 9 & 0.0026 \\
rRun3 & 1 & 20 & 11.1 & 10 & 0.0008 \\
rRun4 & 1 & 50 & 15.6 & 18 & 0.0005 \\
rRun5 & 1 & 80 & 18.7 & 24 & 0.0015 \\
\hline
\end{tabular}

the protoplanet drives gas accretion on to the star. After the central cavity opens up the protoplanet starves the star from gas, with the accretion rate dropping considerably. Mass flows through the inner cavity via accretion streams as in a circumbinary disc cavity (Artymowicz \& Lubow 1996) but accretion happens preferentially on to the protoplanet, the lower mass object of the system as expected: the protoplanet's accretion rate is twice that of the star's (Fig. 24).

\section{THE EFFECT OF THE PROTOPLANET'S ORBITAL RADIUS (WITH RADIATIVE FEEDBACK FROM THE PROTOPLANET/STAR)}

We now examine the evolution of a $1-M_{\mathrm{J}}$ protoplanet placed at different orbital radii within a protostellar disc, including the radiative feedback from the protoplanet and from the central star as described in Section 3.1 (see also Appendix B) [setting $T(1 \mathrm{au})=$ $250 \mathrm{~K}$ for the pseudo-background temperature due to the central star]. These simulations (see Table 3, Fig. 25) probably represent more realistically the evolution of a protoplanet within a young disc. We use the Semenov et al. (2003) opacities for this set of runs.

The semimajor axis evolution of the protoplanet is shown in Fig. 26. As in the previous runs the protoplanet migrates inwards while opening up a gap (or a cavity, if its initial semimajor axis is small enough, i.e. $<20 \mathrm{au}$ ). As in the previous runs without radiative feedback, a protoplanet that orbits close to the central star $\left(\alpha_{p, i}=5 \mathrm{au}\right)$ migrates slightly outwards. For protoplanets that are initially in the inner disc region, their radiative feedback does not play an important role, and their evolution is quite similar to the case without any feedback, as presented in Section 6. The edges of the gap opened up by the protoplanet are already hot and gravitationally stable; therefore, the protoplanet continues to migrate inwards but at a slower pace (Fig. 27). On the other hand, radiative feedback from the protoplanet plays an important role for planets that are initially in the outer, relatively cold disc region. This radiative feedback heats the gap edges, stabilizing them and ensuring that the protoplanets continue to migrate inwards in contrast to the case without radiative feedback, in which, after the gap opens up, the protoplanet migrate outwards.

Similarly to the previous case, we find that interaction with the gravitationally stable gap edges suppresses excessive mass growth so that the protoplanet's mass is near the brown dwarf-planet limit (Fig. 28). Protoplanets that form in the inner disc region find themselves in the inner cavity around the star and their masses are just below the deuterium-burning limit. Protoplanets that form further away from the star go through a longer gap-opening phase as they migrate within the gas-rich disc and they accrete more mass, so that their final mass at the end of the simulation is just above the deuterium-burning limit. Due to this longer gap-opening phase, protoplanets in the outer disc region show a strong peak in their accretion rates (Fig. 29, top) and high corresponding luminosities, which at the earliest phases of the protoplanets' evolution can reach a few tenths of the solar luminosity (Fig. 29, bottom).

The eccentricity of the protoplanet orbit initially grows during the gap opening phase, but thereafter it quickly dampens so that protoplanets at any given radii end up with nearly circular orbits (see Fig. 30). We find that interactions with a gravitationally stable disc dampen the eccentricity effectively.

The accretion rate on to the central star is sensitive to the position of the protoplanet in the disc (Fig. 31, top). When the protoplanet is in the inner disc region, it starves off the young star so that the accretion rate on to it is only half the accretion on to the protoplanet (Fig. 31, bottom). When the protoplanet is in the outer disc region, the accretion on to the central star is heavily suppressed during the gap opening phase, whereas it is enhanced significantly immediately afterwards and for a few thousand years (Fig. 31, bottom). However, in all cases the accretion rate on to the protoplanet is higher than the accretion rate on to the star at the end of the simulation.

We conclude that radiative feedback from the protoplanet plays an important role for its orbital evolution and its mass growth only for protoplanets that are formed in outer cold region of a protoplanetary disc. Such protoplanets may have formed by gravitational fragmentation during an early stage of the disc's formation and evolution, while these discs are still relatively massive (e.g. MacFarlane \& Stamatellos 2017).

\section{SUMMARY AND DISCUSSION}

The final fate of a planet that forms in a protostellar disc is determined by how the two interact with each other. Disc-planet interactions are more critical if the planet forms early on during its parent disc evolution, while this disc is relatively massive and possibly non-axisymmetric. The effect of the disc is two-fold: (i) it exchanges angular momentum with the planet, to facilitate migration (inward or outward), (ii) it exchanges mass with the planet allowing it to grow (but the opposite may also be possible; see Nayakshin 2017a).

Here, we presented simulations of the evolution of a Jupiter-like planet-seed in a relatively massive disc $\left(0.1 \mathrm{M}_{\odot}\right)$ around a Sun-like star $\left(1 \mathrm{M}_{\odot}\right)$, expanding on the work of Stamatellos (2015). The disc is massive enough for its gravity to play an important role in the system's evolution, but not massive enough to fragment due to the development of gravitational instabilities $(Q \gtrsim 1.5)$. We do not restrict the formation mechanism of the planetary seed, although it is rather unlikely that such a protoplanet may have formed by core accretion early on during the disc's lifetime (i.e. within several $10^{4} \mathrm{yr}$ ). On the other hand, such large planet-seeds form naturally fast (on a dynamical time-scale) by disc fragmentation. Nevertheless, the results of this paper are independent of the assumed planet formation scenario.

The evolution of such a planet-seed (protoplanet) is followed for a relatively short duration corresponding to the disc lifetime (20 kyr; see Appendix C for the long-term evolution) but even within such a short time-scale disc-planet interactions are important for determining the properties of the protoplanet. It has been suggested that the strong nature of the planet-disc interactions and the inability of the planet to open up a gap in such a massive disc, may lead to rapid inward migration (Baruteau et al. 2011; Michael et al. 2011; Malik et al. 2015) and the demise of the planet as it falls on to its parent star. The findings of our simulations contradict the results of these studies as we find that the protoplanet is able to open up a gap in the disc so that its fast inward migration is halted (as also pointed 


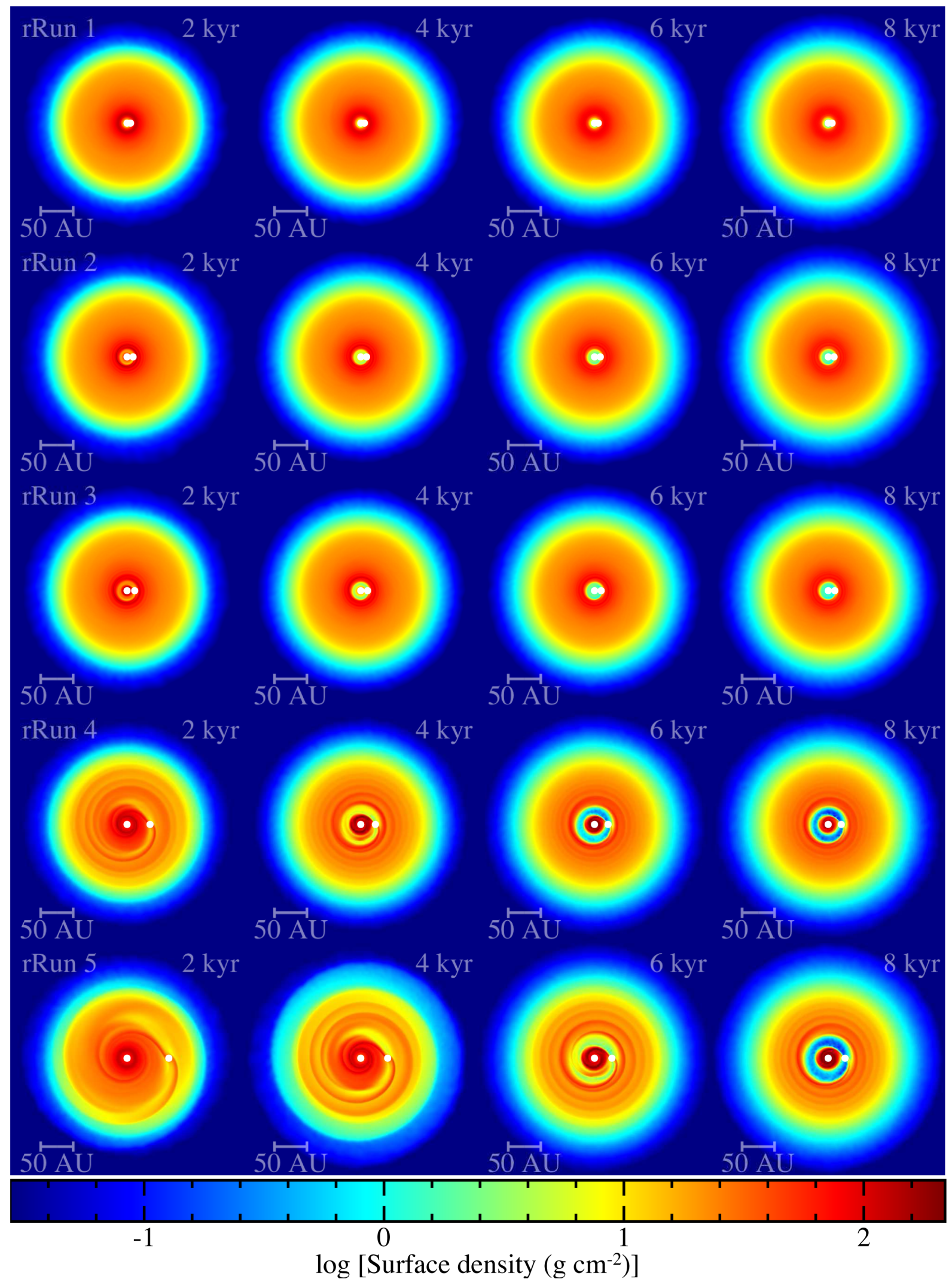

Figure 25. The evolution of the disc surface density in the five simulations in which the radiative feedback from the protoplanet and the star are included (see Table 3). The disc is stable due to the feedback provided by the protoplanet. The protoplanet generally migrates inwards, creating a cavity around the central star (see discussion in the text). 


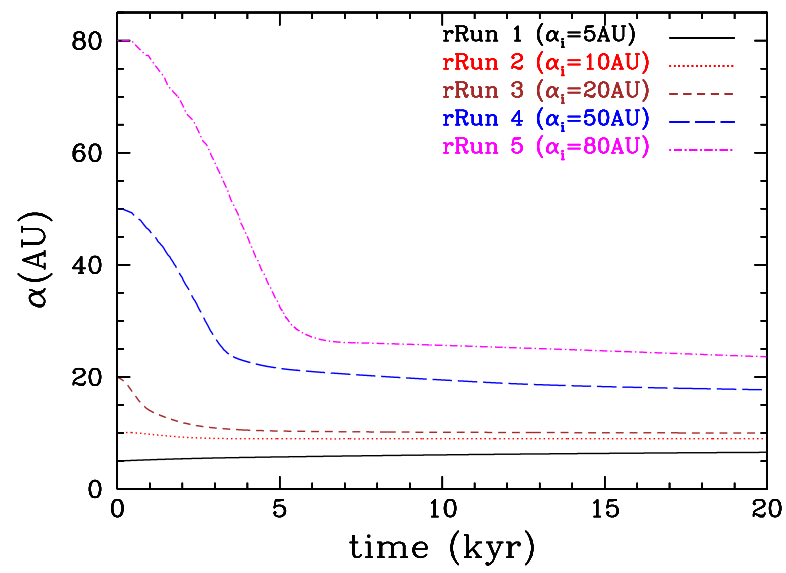

Figure 26. The semimajor axis evolution of an initially $1-M_{\mathrm{J}}$ protoplanet in a $0.1-\mathrm{M}_{\odot}$ disc, including the radiative feedback from the protoplanet. The protoplanet migrates inwards opening up a gap. Once the gap opens up, the migration slows down. There is no outward migration irrespective of the orbital radius as the protoplanet's radiation stabilizes the gap edges.

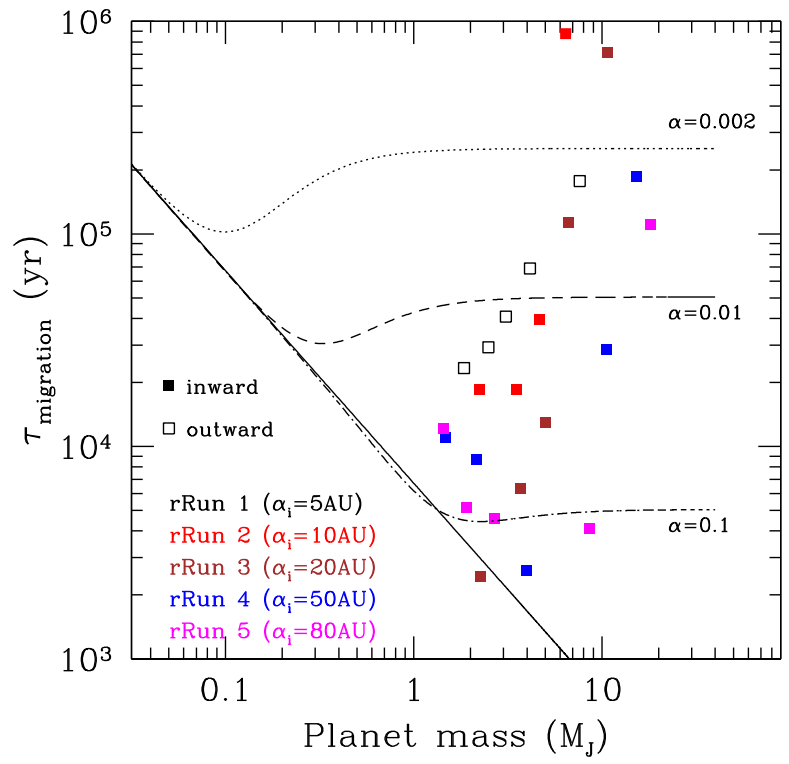

Figure 27. The migration time-scales of protoplanets placed at different orbital radii within a protostellar disc, in the runs where both radiative feedback from the protoplanet and the star are included. Protoplanets migrate inwards, initially on a Type I migration time-scale $\left(\sim 10^{4} \mathrm{yr}\right)$ and once the gap is opened up on a Type II migration time-scale $\left(\sim 10^{5} \mathrm{yr}\right)$. Lines correspond to analytical calculations as in Fig. 8. Filled boxes correspond to inward migration and empty boxes to outward migration.

out in Stamatellos 2015). However, we encounter an alternative problem: the protoplanet grows in mass by accreting gas from the disc, so that in most cases it becomes a brown dwarf (Stamatellos \& Whitworth 2009a; Stamatellos \& Whitworth 2011; Kratter et al. 2010; Zhu et al. 2012). The final state of the planet depends on where it has formed in the disc, but also on the physics igredients that are at play during its evolution. More specifically, this work demonstrates that radiative heating from the young accreting protoplanet plays an critical role; this radiative feedback is currently not well understood nor constrained (Marleau et al. 2017; Szulágyi \& Mordasini 2017). The main results of this work are discussed and placed in a wider context in the following subsections.

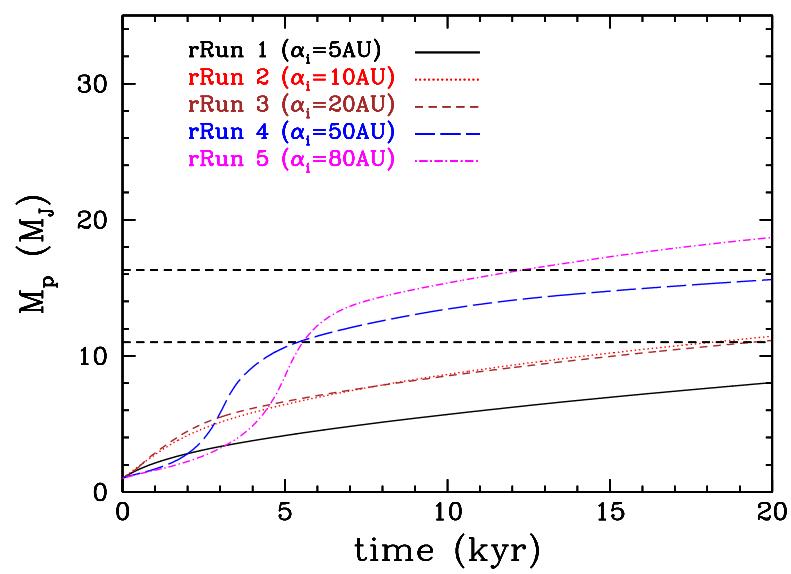

Figure 28. Mass growth of a $1-M_{\mathrm{J}}$ protoplanet placed at different radii within a protostellar disc, when the radiative feedback from the protoplanet and the star are included. The mass growth of the protoplanet is suppressed and the protoplanet remains within the planetary-mass regime even if it has formed in the outer disc region. The horizontal dashed lines correspond to the deuterium-burning mass-limit.
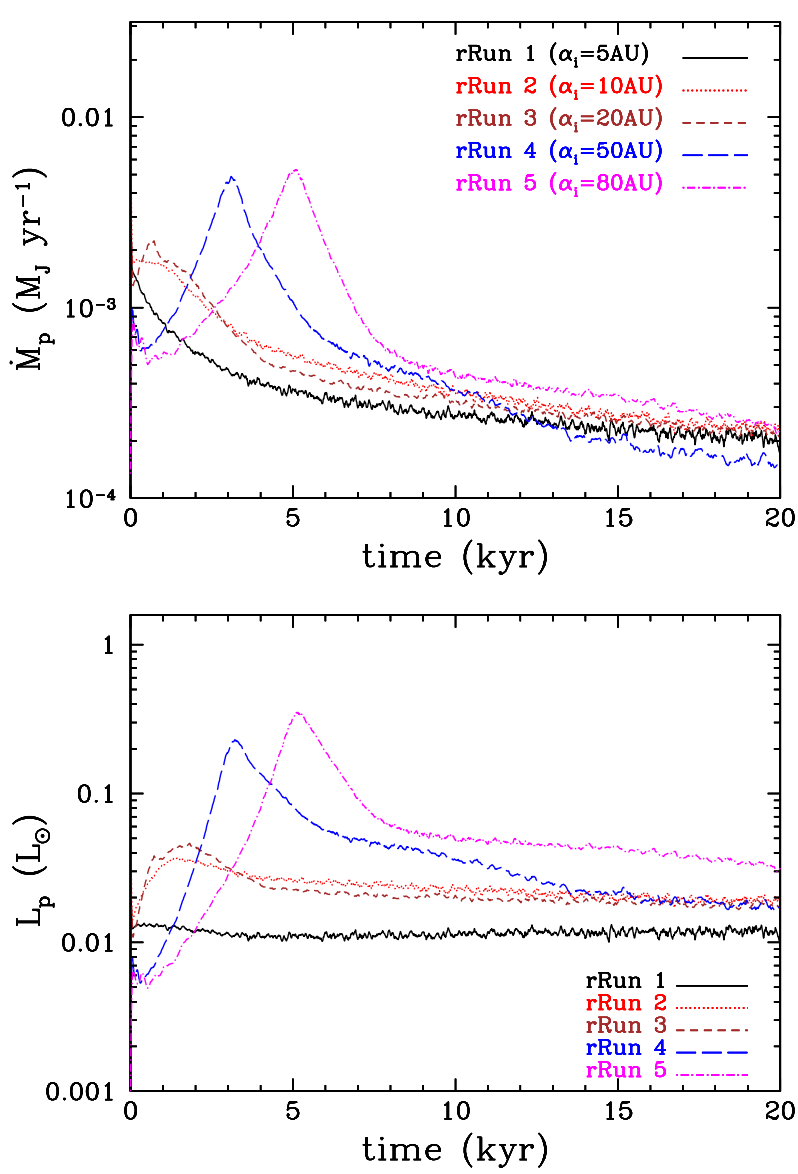

Figure 29. Accretion rate (top) on to a $1-M_{\mathrm{J}}$ protoplanet placed at different radii within a protostellar disc, and its corresponding accretion luminosity (bottom), in the runs where both radiative feedback from the protoplanet and the star are included. The protoplanets during the gap opening phase exhibit high accretion rates and corresponding accretion luminosities. (Note that in this set of runs the protoplanet's luminosity is fed back into the disc.) 


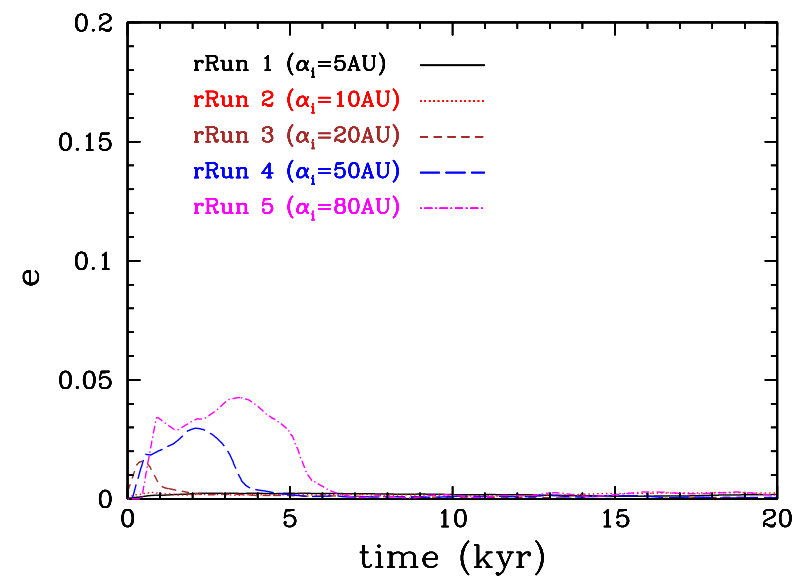

Figure 30. Eccentricity of a $1-M_{\mathrm{J}}$ protoplanet placed at different radii within a protostellar disc, in the runs where both radiative feedback from the protoplanet and the star are included. The eccentricity increases during the gap opening phase but thereafter quickly dampens and the orbit of the protoplanet becomes circular.

\subsection{Protoplanet mass growth}

Gas accretion is significant during the initial stages of the protoplanet's evolution, during the gap-opening phase. In most of the simulations presented here, the protoplanet grows to the planetbrown dwarf mass-limit $\left(\sim 13 M_{\mathrm{J}}\right)$ within a few thousand years. Once the gap is opened up, accretion occurs mainly from inside the protoplanet's orbit. However, a fraction of it is also accreted from outside the protoplanet's orbit; when the gap edges are gravitationally unstable (low Toomre- $Q$, i.e. $Q \lesssim 2$ ), then an almost equal amount of gas is accreted from outside the protoplanet's orbit. The accretion rate on to the protoplanet is higher for higher viscosity, and for more efficient cooling (note that cooling is modulated by the opacity). The role of radiation feedback from the protoplanet is critical as it considerably decreases the accretion rate of gas on to it and therefore its final mass. The effect of radiation feedback from the central star is rather minimal (Appendix B). We also found a dependence of the protoplanet's final mass on its initial position within the disc: a planet that is relatively closer to the central star $(\lesssim 20 \mathrm{au})$ quickly opens up a cavity around its parent star and resides near the outer edge of this cavity. In this case, the star-protoplanet system behaves as a low mass ratio binary system attended by a circumbinary disc. In such a system, gas flows to the star and the protoplanet only through streams that pass through the Lagrangian points of the system (Artymowicz \& Lubow 1994; Artymowicz \& Lubow 1996), and therefore accretion is slow. These are the only cases (with or without radiative feedback from the protoplanet) that the protoplanet's mass remains within the planetary-mass regime at the end of the simulations. As the mass-loss of the disc due to accretion on to the star and the protoplanet is lower in this case, the disc is expected to live longer, allowing other processes, e.g. photoevaporation (Alexander et al. 2006; Owen et al. 2010) or disc winds (Suzuki \& Inutsuka 2009, 2014; Suzuki, Muto \& Inutsuka 2010; Bai \& Stone 2013; Fromang et al. 2013; Lesur, Ferreira \& Ogilvie 2013; Gressel et al. 2015; Bai 2016), to disperse the disc fast enough for the protoplanet to survive as a planet, not a brown dwarf. Another way to suppress mass growth is to eject the protoplanet from the disc (in multiple-planet systems) so that it ends up as a free-floating planet (Li et al. 2015, 2016; Mercer \& Stamatellos 2017).
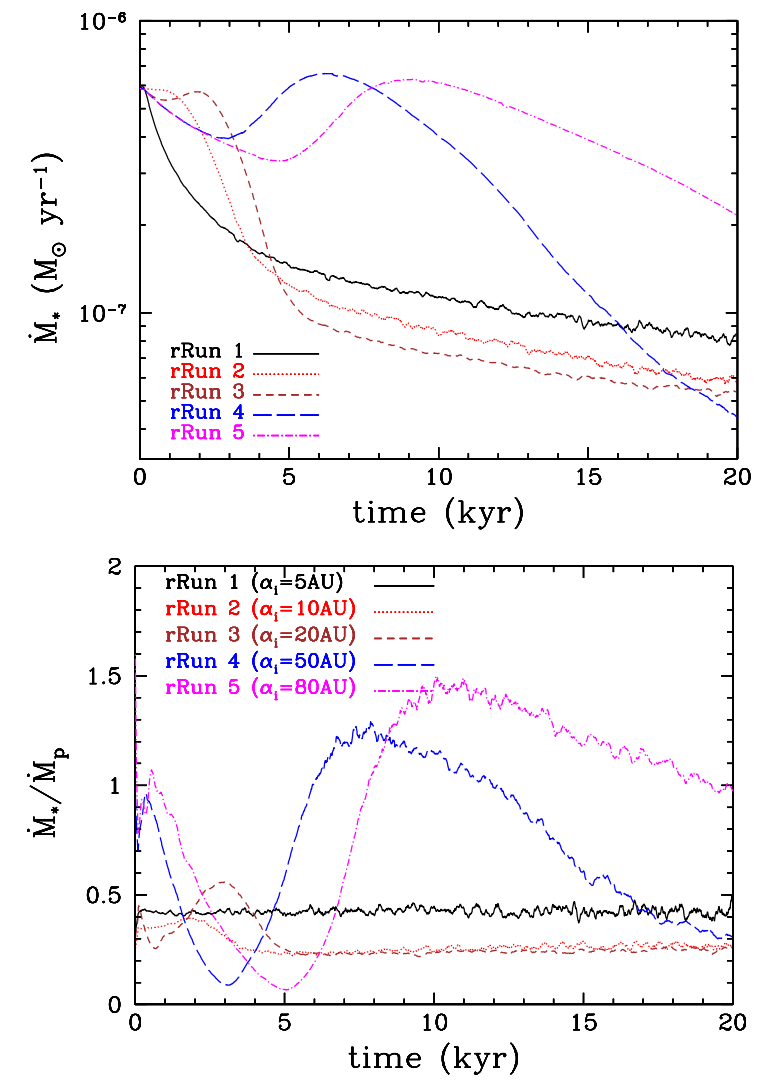

Figure 31. The accretion rate on to the central star (top) and the ratio of the accretion rate on to the star to the accretion rate on to the protoplanet (bottom), in the runs where both radiative feedback from the protoplanet and the star are included. The accretion rate on to the star is lower when the protoplanet is in the inner disc region.

\subsection{Protoplanet migration}

Disc-planet interactions result in an initial phase of fast inward migration that lasts for a few thousand years, apart from the runs in which the protoplanet is very close to its parent star $(\lesssim 10 \mathrm{au})$; in these runs, the protoplanet's orbit changes only slightly as it quickly opens up a cavity around the star. The migration time-scale for this initial phase is $\sim 10^{4} \mathrm{yr}$, i.e. similar to the Type I migration timescale established for planet migration in low-mass discs. This is consistent with previous studies (Baruteau et al. 2011; Michael et al. 2011; Malik et al. 2015). However, we find that the protoplanet is always able to open up a gap in the disc, and thereafter the migration pattern diverges from that in previous studies.

We find that if the gap edges are gravitationally unstable (low Toomre- $Q$, i.e. $Q \lesssim 2$ ), then the protoplanet starts migrating outwards on a time-scale $\sim 10^{5} \mathrm{yr}$, as a high fraction of the gas it accretes (almost about 50 per cent) is higher angular momentum gas from outside its orbit. If the gap edges are gravitationally stable (i.e. in the runs with protoplanet radiative feedback, or when the protoplanet is closer to the star, in a hotter disc region) then the migration continues to be inwards but on a longer, Type II migration time-scale, i.e. $\sim 10^{5} \mathrm{yr}$, with the planet accreting mainly lower angular momentum gas from inside its orbit.

Contrary to previous studies we have allowed the protoplanet to grown in mass, and, critically, we have used a more sophisticated treatment of the radiative transfer rather than the commonly used $\beta$-cooling approximation (Baruteau et al. 2011; Malik et al. 2015). The method we use allows the gas to modulate its cooling/heating 
depending on its properties (density, temperature); this enables gap opening and facilitates enhanced mass growth of the circumplanetary disc and of the planet.

In the present calculations, this 'accretion from outside' that is responsible for initiating outward migration is driven by the gravitational instability in the outer disc. However, it is possible this may be promoted by any mechanism that makes large amplitude fluctuations in the outer disc, such as the magnetorotational instability (but see Gressel, Nelson \& Turner 2012) or other instabilities.

\subsection{Protoplanet eccentricity}

The eccentricity of a protoplanet grows up to 0.05 during the initial gap opening phase. Thereafter, two patterns are seen: protoplanets that reside within a gap with gravitationally unstable gap edges show further eccentricity growth up to $0.1-0.2$, whereas the eccentricity of protoplanets within a gap with stable edges dampens, making their orbits nearly circular. The eccentricity growth is not monotonic but shows a stochastic pattern, characteristic of both periodic and random interactions with condensations present near the gap edges. In the long-term runs $\left(10^{5} \mathrm{yr}\right)$, we see further eccentricity growth up to $\sim 0.3$ (see Appendix C). Almost in all cases that we examined here, we see a minimum eccentricity growth of a few 0.01 which may provide the seed for further eccentricity growth (Goldreich \& Sari 2003; Duffell \& Dong 2015). Therefore, early protoplanet interactions with a massive but gravitationally stable disc may explain in some cases the high eccentricities of giant planets in single-planet systems (Wright et al. 2011; Dunhill \& Stamatellos 2018). On the other hand, we see that protoplanets ending up in the inner disc region ( $\lesssim 20 \mathrm{au}$ ), tend to have circular orbits.

\subsection{Effect on the parent star}

We find that in most cases the accretion rate on to the protoplanet is higher or at comparable to the accretion rate on to the star. If the protoplanet is close enough to the central star, it creates a cavity, resides within it (at its outer edge), and seemingly starves off the central star from gas, accreting most of the incoming gas. This stage shows similarities with the transition disc phase (Espaillat et al. 2014).

\subsection{Observability of protoplanets}

The luminosity of a protoplanet can be quite high during the initial stages of its evolution, as the accretion rate on it is relatively high (typically $\sim 10^{-3} M_{\mathrm{J}} \mathrm{yr}^{-1}$, but could be up to 10 times higher during the gap opening phase). Assuming a relatively large radius for the protoplanet of $R_{\text {acc }}=3 \mathrm{R}_{\odot}$ (which is expected for a protoplanet that has formed by disc fragmentation), we find a typical accretion luminosity of a few $0.1 \mathrm{~L}_{\odot}$ (see also Inutsuka et al. 2010). Combined with the fact that the protoplanet starves off its central star from gas, it may make its detection easier.

The high accretion phase lasts only for a few $10^{3}-10^{4} \mathrm{yr}$, and therefore only a small fraction of transition discs (assuming that they all are due to planetary companions) are expected to be seen in this high-luminosity phase. If transition discs live for $1 \mathrm{Myr}$, then, from time-scale arguments, we suggest that only a small percentage, 0.01 per cent to 0.1 per cent, of transition discs may have observable, high-luminosity protoplanets (or brown dwarfs).

\subsection{Circumplanetary discs}

Protoplanets are attended by circumplanetary discs that are fed with gas from the circumstellar disc. We find that these discs are hotter than their surroundings and therefore easier to observe. Their temperatures are a few hundred Kelvin and they are hotter when radiative feedback from the protoplanet is taken into account. If the protoplanet migrates outwards its circumplanetary disc becomes more massive $\left(\sim 1 M_{\mathrm{J}}\right)$ and larger $\sim 10$ au.

A protoplanet could be detected indirectly through its circumplanetary disc (Szulágyi et al. 2017). The circumplanetary disc of a young luminous protoplanet is hotter than in the case of nonluminous protoplanet (see Fig. 12), therefore it would emit significant long-wavelength radiation even if it is not particularly dense. This long-wavelength radiation would escape mostly unattenuated from the system, whereas short-wavelength radiation that is emitted from the protoplanet itself may be significantly attenuated, especially if the protoplanet orbits a young embedded protostar (e.g. in a Class 0 object).

\section{CONCLUSIONS}

Our study shows that the final fate of a Jupiter-like planet-seed formed early-on during the life of a protostellar disc depends both on the initial properties of the seed (e.g. its initial orbit), and the physical processes that are at play (e.g. the role of the protoplanet's accretion luminosity). The protoplanet may end up as a massive giant planet on a circular orbit close to its parent star or as a lowor high- mass brown dwarf on an eccentric wide orbit.

The parameter space has not been fully explored. It is expected that the initial disc mass will play an important role as it will determine to a wide extent whether the disc is close to being gravitationally unstable or not. Additionally, the initial mass of the protoplanet is important; lower mass planet-seeds (i.e. a few tenths of the mass of Jupiter) will grow in mass slower and may have a better chance to survive as planets (Malik et al. 2015; Nayakshin 2017a).

We have assumed that a protoplanet has fully formed (i.e. it is a bound object) at the beginning of the simulation. However, if the planet has formed by disc fragmentation then the formation timescale of the clump and how this evolves to a bound object should be taken into account (Boley et al. 2010; Zhu et al. 2012). Previous studies have shown that a large fraction of these pre-protoplanet clumps may be disrupted, with only a few of them surviving (Boley et al. 2010; Tsukamoto et al. 2015; Hall et al. 2017).

The protoplanets in our simulations are represented by sink particles that accrete gas as this approaches within 0.1 au from them. The use of sinks in hydrodynamic simulations is needed to avoid small time-steps, but it could lead to enhanced gas accretion that may depend on how well the circumplanetary disc is resolved. The study of how the accretion rate on to the protoplanet varies with resolution and with respect to the hydrodynamic method used (e.g. SPH versus grid-based) is important but outside the scope of this paper. However, we note that the accretion rates on to the protoplanets that we obtain in this work are similarly high (within a factor of a few) as the accretion rates obtained from previous studies (D'Angelo \& Lubow 2008; Ayliffe \& Bate 2009; Zhu et al. 2012; Gressel et al. 2013), which use different numerical methods and disc-planet initial setups.

The physical processes involved are also important. We have shown that the disc thermodynamics, i.e. how fast the disc heats and cools, affects the accretion rate on to the protoplanet (see also Nayakshin 2017b), its ability to open up a gap in the disc, and its final mass and orbital radius. The $\beta$-cooling approximation (e.g. Baruteau et al. 2011), in which the cooling rate is proportional to the local orbital period, does not properly capture the thermodynamics of the circumstellar and circumplanetary disc, and may underestimate the mass flow on to the protoplanet and its ability to open up 
a gap. We also found that the protoplanet accretion luminosity is significant in shaping the properties of the disc in the vicinity of the planet and may stabilize the edges of the gap in which the planet resides, altering its migration pattern.

Therefore, there are many uncertainties regarding the final fate of a young protoplanet that need to be taken into account in population synthesis models (e.g. Mordasini, Alibert \& Benz 2009; Forgan \& Rice 2013; Nayakshin 2017a) in order for numerical results on planet formation at an early phase during the protostellar disc evolution to be compared with the observed properties of exoplanets. The observational evidence that only 1-10 per cent of star hosts gas giant planets on wide orbits (Brandt et al. 2014; Galicher et al. 2016; Vigan et al. 2017), corresponds to the final outcome of the planet formation process which does not necessarily reflect the properties of newly formed planet-seeds in young protostellar discs.

\section{ACKNOWLEDGEMENTS}

We thank the referee for carefully reading the original manuscript and for providing many suggestions that have improved the paper. DS thanks Sergei Nayakshin, Alex Dunhill, and Anthony Mercer for useful discussions. Simulations were performed using the UCLAN High Performance Computing (HPC) facility and the COSMOS Shared Memory system at DAMTP, University of Cambridge operated on behalf of the Science and Technology Facilities Council (STFC) Distributed Research utilizing Advanced Computing (DiRAC) HPC Facility. This equipment is funded by the Department for Business, Innovations \& Skills (BIS) National E-infrastructure capital grant ST/J005673/1 and STFC grants ST/H008586/1, ST/K00333X/1. SEREN has been developed and maintained by David Hubber, who we thank for his help. Surface density plots were produced using SPLASH (Price 2007). DS acknowledges support from STFC Grant ST/M000877/1. This work was supported from a Royal Society-Daiwa Foundation International Exchanges award between UCLAN and Nagoya University.

\section{REFERENCES}

Alexander R. D., Clarke C. J., Pringle J. E., 2006, MNRAS, 369, 216

Alexander R., Pascucci I., Andrews S., Armitage P., Cieza L., 2014, in Beuther H., Klessen R., Dullemond C., Henning T., eds, Protostars and Planets VI. Univ. Arizona Press, Tucson, AZ

Aller K. M. et al., 2013, ApJ, 773, 63

ALMA Partnership et al., 2015, ApJ, 808, L3

Andrews S. M., Williams J. P., 2007, ApJ, 659, 705

Andrews S. M., Wilner D. J., Hughes A. M., Qi C., Dullemond C. P., 2009, ApJ, 700, 1502

Andrews S. M. et al., 2016, ApJ, 820, L40

Artymowicz P., Lubow S. H., 1994, ApJ, 421, 651

Artymowicz P., Lubow S. H., 1996, ApJ, 467, L77

Ayliffe B. A., Bate M. R., 2009, MNRAS, 393, 49

Bai X.-N., 2016, ApJ, 821, 80

Bai X.-N., Stone J. M., 2013, ApJ, 767, 30

Bailey V. et al., 2014, ApJ, 780, L4

Baraffe I., Elbakyan V. G., Vorobyov E. I., Chabrier G., 2017, A\&A, 597, A19

Baruteau C., Meru F., Paardekooper S.-J., 2011, MNRAS, 416, 1971

Bate M. R., 2014, MNRAS, 442, 285

Bate M. R., Bonnell I. A., Price N. M., 1995, MNRAS, 277, 362

Bate M. R., Lubow S. H., Ogilvie G. I., Miller K. A., 2003, MNRAS, 341, 213

Bell K. R., Lin D. N. C., 1994, ApJ, 427, 987

Benítez-Llambay P., Masset F., Koenigsberger G., Szulágyi J., 2015, Nature, 520, 63

Bodenheimer P., Pollack J. B., 1986, Icarus, 67, 391

Boley A. C., 2009, ApJ, 695, L53
Boley A. C., Mejía A. C., Durisen R. H., Cai K., Pickett M. K., D’Alessio P., 2006, ApJ, 651, 517

Boley A. C., Hayfield T., Mayer L., Durisen R. H., 2010, Icarus, 207, 509

Boss A. P., 1988, ApJ, 331, 370

Boss A. P., 1997, Science, 276, 1836

Boyd D. F. A., Whitworth A. P., 2005, A\&A, 430, 1059

Brandt T. D. et al., 2014, ApJ, 794, 159

Cai K., Durisen R. H., Boley A. C., Pickett M. K., Mejía A. C., 2008, ApJ, 673, 1138

Cameron A. G. W., 1978, Moon Planets, 18, 5

Cloutier R., Lin M.-K., 2013, MNRAS, 434, 621

Crida A., Morbidelli A., 2007, MNRAS, 377, 1324

Crida A., Morbidelli A., Masset F., 2006, Icarus, 181, 587

D'Angelo G., Lubow S. H., 2008, ApJ, 685, 560

Dipierro G., Price D., Laibe G., Hirsh K., Cerioli A., Lodato G., 2015, MNRAS, 453, L73

Duffell P. C., Dong R., 2015, ApJ, 802, 42

Duffell P. C., Haiman Z., MacFadyen A. I., D’Orazio D. J., Farris B. D., 2014, ApJ, 792, L10

Dunhill A. C., Stamatellos D., 2018, A\&A, submitted

Dürmann C., Kley W., 2015, A\&A, 574, A52

Dürmann C., Kley W., 2017, A\&A, 598, A80

Espaillat C. et al., 2014, in Beuther H., Klessen R., Dullemond C., Henning T., eds, Protostars and Planets VI. Univ. Arizona Press, Tucson, AZ, p. 497

Faherty J. K., Burgasser A. J., Cruz K. L., Shara M. M., Walter F. M., Gelino C. R., 2009, AJ, 137, 1

Forgan D., Rice K., 2011, MNRAS, 417, 1928

Forgan D., Rice K., 2013, MNRAS, 432, 3168

Forgan D., Rice K., Stamatellos D., Whitworth A. P., 2009, MNRAS, 394, 882

Fromang S., Latter H., Lesur G., Ogilvie G. I., 2013, A\&A, 552, A71

Fujii Y. I., Okuzumi S., Tanigawa T., Inutsuka S.-i., 2014, ApJ, 785, 101

Galicher R. et al., 2014, A\&A, 565, L4

Galicher R. et al., 2016, A\&A, 594, A63

Gammie C. F., 2001, ApJ, 553, 174

Gárate M., Cuadra J., Montesinos M., 2017, MNRAS, preprint (arXiv:1711.01372)

Goldreich P., Sari R., 2003, ApJ, 585, 1024

Goldreich P., Ward W. R., 1973, ApJ, 183, 1051

Gonzalez J.-F., Laibe G., Maddison S. T., Pinte C., Ménard F., 2015, MNRAS, 454, L36

Greaves J. S., Richards A. M. S., Rice W. K. M., Muxlow T. W. B., 2008, MNRAS, 391, L74

Gressel O., Nelson R. P., Turner N. J., 2012, MNRAS, 422, 1140

Gressel O., Nelson R. P., Turner N. J., Ziegler U., 2013, ApJ, 779, 59

Gressel O., Turner N. J., Nelson R. P., McNally C. P., 2015, ApJ, 801, 84

Haisch K. E., Jr, Lada E. A., Lada C. J., 2001, ApJ, 553, L153

Hall C., Forgan D., Rice K., 2017, MNRAS, 470, 2517

Hernández J., Hartmann L., Calvet N., Jeffries R. D., Gutermuth R., Muzerolle J., Stauffer J., 2008, ApJ, 686, 1195

Hubber D. et al., 2011a, Astrophysics Source Code Library, record ascl:1102.010

Hubber D. A., Batty C. P., Mcleod A., Whitworth A. P., 2011b, A\&A, 529, A27

Inutsuka S., Machida M. N., Matsumoto T., 2010, ApJ, 718, L58

Ireland M. J., Kraus A. L., Martinache F., Law N., Hillenbrand L. A., 2011, ApJ, 726, 113

Isella A. et al., 2016, Phys. Rev. Lett., 117, 251101

Johnson B. M., Gammie C. F., 2003, ApJ, 597, 131

Kratter K. M., Murray-Clay R. A., Youdin A. N., 2010, ApJ, 710, 1375

Kraus A. L., Ireland M. J., Martinache F., Lloyd J. P., 2008, ApJ, 679, 762

Kraus A. L., Ireland M. J., Cieza L. A., Hinkley S., Dupuy T. J., Bowler B. P., Liu M. C., 2014, ApJ, 781, 20

Kuiper G. P., 1951, Natl. Acad. Sci., 37, 1

Kuzuhara M., Tamura M., Ishii M., Kudo T., Nishiyama S., Kandori R., 2011, AJ, 141, 119

Kuzuhara M. et al., 2013, ApJ, 774, 11

Larson R. B., 1969, MNRAS, 145, 271 
Lesur G., Ferreira J., Ogilvie G. I., 2013, A\&A, 550, A61

Li Y., Kouwenhoven M. B. N., Stamatellos D., Goodwin S. P., 2015, ApJ, 805,116

Li Y., Kouwenhoven M. B. N., Stamatellos D., Goodwin S. P., 2016, ApJ, 831,166

Lin M.-K., Papaloizou J. C. B., 2012, MNRAS, 421, 780

Lombardi J. C., McInally W. G., Faber J. A., 2015, MNRAS, 447, 25

Low C., Lynden-Bell D., 1976, MNRAS, 176, 367

Lubow S. H., D’Angelo G., 2006, ApJ, 641, 526

MacFarlane B. A., Stamatellos D., 2017, MNRAS, 472, 3775

Machida M. N., Inutsuka S.-i., Matsumoto T., 2006, ApJ, 649, L129

Machida M. N., Inutsuka S., Matsumoto T., 2010, ApJ, 724, 1006

Machida M. N., Inutsuka S., Matsumoto T., 2011a, PASJ, 63, 555

Machida M. N., Inutsuka S., Matsumoto T., 2011b, ApJ, 729, 42

Machida M. N., Inutsuka S.-i., Matsumoto T., 2014, MNRAS, 438, 2278

Malik M., Meru F., Mayer L., Meyer M., 2015, ApJ, 802, 56

Marleau G.-D., Klahr H., Kuiper R., Mordasini C., 2017, ApJ, 836, 221

Marley M. S., Fortney J. J., Hubickyj O., Bodenheimer P., Lissauer J. J., 2007, ApJ, 655, 541

Marois C., Macintosh B., Barman T., Zuckerman B., Song I., Patience J., Lafrenière D., Doyon R., 2008, Science, 322, 1348

Masunaga H., Inutsuka S., 1999, ApJ, 510, 822

Masunaga H., Inutsuka S., 2000, ApJ, 531, 350

Matzner C. D., Levin Y., 2005, ApJ, 628, 817

Mercer A., Stamatellos D., 2017, MNRAS, 465, 2

Michael S., Durisen R. H., Boley A. C., 2011, ApJ, 737, L42

Mizuno H., 1980, Prog. Theor. Phys., 64, 544

Montesinos M., Cuadra J., Perez S., Baruteau C., Casassus S., 2015, ApJ, 806,253

Mordasini C., 2013, A\&A, 558, A113

Mordasini C., Alibert Y., Benz W., 2009, A\&A, 501, 1139

Mordasini C., Marleau G.-D., Mollière P., 2017, A\&A, 608, A72

Morris J. P., Monaghan J. J., 1997, J. Comput. Phys., 136, 41

Muzerolle J., Allen L. E., Megeath S. T., Hernández J., Gutermuth R. A., 2010, ApJ, 708, 1107

Naud M.-E. et al., 2014, ApJ, 787, 5

Nayakshin S., 2017a, PASA, 34, e002

Nayakshin S., 2017b, MNRAS, 470, 2387

Nayakshin S., Cha S.-H., 2013, MNRAS, 435, 2099

Nelson R. P., Papaloizou J. C. B., Masset F., Kley W., 2000, MNRAS, 318, 18

Offner S. S. R., Klein R. I., McKee C. F., Krumholz M. R., 2009, ApJ, 703, 131

Ormel C. W., Kuiper R., Shi J.-M., 2015a, MNRAS, 446, 1026

Ormel C. W., Shi J.-M., Kuiper R., 2015b, MNRAS, 447, 3512

Osterloh M., Beckwith S. V. W., 1995, ApJ, 439, 288

Owen J. E., 2014, ApJ, 789, 59

Owen J. E., Ercolano B., Clarke C. J., Alexander R. D., 2010, MNRAS, 401,1415

Pérez L. M. et al., 2016, Science, 353, 1519

Pollack J. B., Hubickyj O., Bodenheimer P., Lissauer J. J., Podolak M., Greenzweig Y., 1996, Icarus, 124, 62

Price D. J., 2007, PASA, 24, 159

Rafikov R. R., 2005, ApJ, 621, L69

Rameau J. et al., 2013, ApJ, 772, L15

Rees M. J., 1976, MNRAS, 176, 483

Rice W. K. M., Lodato G., Armitage P. J., 2005, MNRAS, 364, L56

Rogers P. D., Wadsley J., 2012, MNRAS, 423, 1896

Safronov V. S., 1972, inSafronov V. S., ed., Evolution of the Protoplanetary Cloud and Formation of the Earth and Planets. Translated from Russian. Israel Program for Scientific Translations, Keter Publishing House, Jerusalem, p. 212

Satsuka T., Tsuribe T., Tanaka S., Nagamine K., 2017, MNRAS, 465, 986

Semenov D., Henning T., Helling C., Ilgner M., Sedlmayr E., 2003, A\&A, 410,611

Silk J., 1977, ApJ, 214, 152

Spiegel D. S., Burrows A., Milsom J. A., 2011, ApJ, 727, 57

Stamatellos D., 2015, ApJ, 810, L11

Stamatellos D., Whitworth A. P., 2008, A\&A, 480, 879
Stamatellos D., Whitworth A. P., 2009a, MNRAS, 392, 413

Stamatellos D., Whitworth A. P., 2009b, MNRAS, 400, 1563

Stamatellos D., Whitworth A., 2011, in Martin E. L., Ge J., Lin W., eds, EPJ Web Conf., 16, 05001

Stamatellos D., Whitworth A. P., Bisbas T., Goodwin S., 2007, A\&A, 475, 37

Stamatellos D., Maury A., Whitworth A., André P., 2011a, MNRAS, 413, 1787

Stamatellos D., Whitworth A. P., Hubber D. A., 2011b, ApJ, 730, 32

Stamatellos D., Whitworth A. P., Hubber D. A., 2012, MNRAS, 427, 1182

Suzuki T. K., Inutsuka S.-i., 2009, ApJ, 691, L49

Suzuki T. K., Inutsuka S.-i., 2014, ApJ, 784, 121

Suzuki T. K., Muto T., Inutsuka S.-i., 2010, ApJ, 718, 1289

Szulágyi J., 2017, ApJ, 842, 103

Szulágyi J., Mordasini C., 2017, MNRAS, 465, L64

Szulágyi J., van der Plas G., Meyer M. R., Pohl A., Quanz S. P., Mayer L., Daemgen S., Tamburello V., 2017, MNRAS, preprint (arXiv:1709.04438)

Takahashi S. Z., Inutsuka S.-i., 2014, ApJ, 794, 55

Takahashi S. Z., Tsukamoto Y., Inutsuka S., 2016a, MNRAS, 458, 3597

Takahashi S. Z., Tomida K., Machida M. N., Inutsuka S.-i., 2016b, MNRAS, 463,1390

Tanaka H., Takeuchi T., Ward W. R., 2002, ApJ, 565, 1257

Testi L. et al., 2015, ApJ, 812, L38

Tobin J. J. et al., 2016, ApJ, 818, 73

Tomida K., Tomisaka K., Matsumoto T., Hori Y., Okuzumi S., Machida M. N., Saigo K., 2013, ApJ, 763, 6

Toomre A., 1964, ApJ, 139, 1217

Tsukamoto Y., Takahashi S. Z., Machida M. N., Inutsuka S., 2015, MNRAS, 446,1175

Vaytet N., Chabrier G., Audit E., Commerçon B., Masson J., Ferguson J., Delahaye F., 2013, A\&A, 557, A90

Vigan A. et al., 2017, A\&A, 603, A3

Vorobyov E. I., Basu S., 2005, ApJ, 633, L137

Vorobyov E. I., Basu S., 2006, ApJ, 650, 956

Ward W. R., 1997, Icarus, 126, 261

Whitworth A. P., Stamatellos D., 2006, A\&A, 458, 817

Whitworth A. P., Bate M. R., Nordlund Å., Reipurth B., Zinnecker H., 2007, in Reipurth B., Jewitt D., Keil K., eds, Protostars and Planets V. Univ. Arizona Press, Tucson, AZ, p. 459

Wilkins D. R., Clarke C. J., 2012, MNRAS, 419, 3368

Wright J. T. et al., 2011, PASP, 123, 412

Young M. D., Bertram E., Moeckel N., Clarke C. J., 2012, MNRAS, 426, 1061

Zhu Z., 2015, ApJ, 799, 16

Zhu Z., Hartmann L., Nelson R. P., Gammie C. F., 2012, ApJ, 746, 110

\section{APPENDIX A: MIGRATION TIME-SCALES AND VELOCITIES FOR EACH SET OF SIMULATIONS}

In this section, we present the migration time-scales and the corresponding migration velocities for the set of runs in Tables 1, 2, and 3 .

Table A1. Migration time-scales for the runs in Table 1 calculated at the times indicated in the table. Negative values correspond to the outward migration.

\begin{tabular}{lccccc}
\hline & $0.7 \mathrm{kyr}$ & $1.5 \mathrm{kyr}$ & $\begin{array}{c}2.5 \mathrm{kyr} \\
\tau_{\text {mig }}(\mathrm{kyr})\end{array}$ & $5 \mathrm{kyr}$ & $18 \mathrm{kyr}$ \\
\hline Run1 & 14 & 0.7 & 44 & -140 & -220 \\
Run2 & 11 & 8 & 2.4 & 38 & 150 \\
Run3 & 17 & 6.8 & 5.6 & -56 & -112 \\
Run4 & 15 & 11 & 0.5 & -71 & -270 \\
Run5 & 12 & 11 & -31 & -27 & -68 \\
\hline
\end{tabular}


Table A2. Migration velocities for the runs in Table 1 calculated at the times indicated in the table. Negative values correspond to the outward migration.

\begin{tabular}{lccccc}
\hline & $0.7 \mathrm{kyr}$ & $1.5 \mathrm{kyr}$ & $\begin{array}{c}2.5 \mathrm{kyr} \\
v_{\text {mig }}\left(\mathrm{au} \mathrm{kyr}^{-1}\right)\end{array}$ & $5 \mathrm{kyr}$ & $18 \mathrm{kyr}$ \\
\hline Run1 & 3.4 & 6.4 & 0.8 & -0.26 & -0.18 \\
Run2 & 4.4 & 5.2 & 15 & 0.5 & 0.12 \\
Run3 & 2.9 & 6.7 & 6.0 & -0.59 & -0.3 \\
Run4 & 3.2 & 4.2 & 7.7 & -0.5 & -0.13 \\
Run5 & 4.1 & 4.2 & -1.4 & -1.6 & -0.8 \\
\hline
\end{tabular}

Table A3. Migration time-scales for the runs in Table 2. These are calculated at the times indicated in the table. Negative values correspond to the outward migration.

\begin{tabular}{lccccc}
\hline & $0.7 \mathrm{kyr}$ & $1.5 \mathrm{kyr}$ & $\begin{array}{c}2.5 \mathrm{kyr} \\
\tau_{\mathrm{mig}}(\mathrm{kyr})\end{array}$ & $5 \mathrm{kyr}$ & $18 \mathrm{kyr}$ \\
\hline sRun1 & -23 & -30 & -43 & -70 & -140 \\
sRun2 & 15 & 16 & 43 & $2.0 \times 10^{3}$ & $2.3 \times 10^{3}$ \\
sRun3 & 2.6 & 6 & 10 & 150 & 400 \\
sRun4 & 12 & 11 & -30 & -27 & -70 \\
sRun5 & 12 & 5.5 & 34 & 41 & -210 \\
\hline
\end{tabular}

Table A4. Migration velocities for the runs in Table 2. These are calculated at the times indicated in the table. Negative values correspond to the outward migration.

\begin{tabular}{lccccc}
\hline & $0.7 \mathrm{kyr}$ & $1.5 \mathrm{kyr}$ & $\begin{array}{c}2.5 \mathrm{kyr} \\
v_{\text {mig }}\left(\mathrm{au} \mathrm{kyr}^{-1}\right)\end{array}$ & $5 \mathrm{kyr}$ & $18 \mathrm{kyr}$ \\
\hline sRun1 & -0.2 & -0.17 & -0.13 & -0.08 & -0.05 \\
sRun2 & 0.7 & 0.6 & 0.21 & 0.005 & 0.004 \\
sRun3 & 6.7 & 2.2 & 1.2 & 0.07 & 0.025 \\
sRun4 & 4.1 & 4.2 & -1.4 & -1.6 & -0.8 \\
sRun5 & 7.0 & 14 & 2.0 & 1.7 & -0.3 \\
\hline
\end{tabular}

Table A5. Migration time-scales for the runs in Table 3. These are calculated at the times indicated in the table. Negative values correspond to the outward migration.

\begin{tabular}{lccccc}
\hline & $0.7 \mathrm{kyr}$ & $1.5 \mathrm{kyr}$ & $\begin{array}{c}2.5 \mathrm{kyr} \\
\tau_{\text {mig }}(\mathrm{kyr})\end{array}$ & $5 \mathrm{kyr}$ & $18 \mathrm{kyr}$ \\
\hline rRun1 & -23 & -29 & -41 & -70 & -180 \\
rRun2 & 19 & 19 & 40 & 880 & $1.4 \times 10^{4}$ \\
rRun3 & 2.4 & 6.3 & 13 & 140 & 710 \\
rRun4 & 11 & 8.7 & 2.6 & 29 & 190 \\
rRun5 & 12 & 5.1 & 4.5 & $4 \mathrm{~d}^{\prime}$ & 110 \\
\hline
\end{tabular}

Table A6. Migration velocities for the runs in Table 3. These are calculated at the times indicated in the table. Negative values correspond to the outward migration.

\begin{tabular}{lccccc}
\hline & $0.7 \mathrm{kyr}$ & $1.5 \mathrm{kyr}$ & $\begin{array}{c}2.5 \mathrm{kyr} \\
v_{\text {mig }}\left(\mathrm{au} \mathrm{kyr}^{-1}\right)\end{array}$ & $5 \mathrm{kyr}$ & $18 \mathrm{kyr}$ \\
\hline rRun1 & -0.21 & -0.18 & -0.13 & -0.008 & -0.04 \\
rRun2 & 0.5 & 0.5 & 0.2 & 0.01 & $6 \times 10^{4}$ \\
rRun3 & 6.9 & 2.1 & 0.9 & 0.09 & 0.014 \\
rRun4 & 4.5 & 5.0 & 13 & 0.8 & 0.1 \\
rRun5 & 6.6 & 15.0 & 15.0 & 8.4 & 0.2 \\
\hline
\end{tabular}

\section{APPENDIX B: THE EFFECT OF RADIATION FROM THE CENTRAL STAR ON THE DIRECTION OF MIGRATION}

The effect of radiative feedback from the protoplanet is important only when the energy that is fed back to the disc alters its dynamical state. In this section, we examine how important radiative feedback from the protoplanet is, when the central star is also heating the disc.

We compare five simulations of an initially $1-M_{\mathrm{J}}$ protoplanet placed at 50 au from the central star in a $0.1-\mathrm{M}_{\odot}$ disc: (i) without any radiative feedback (Run 1; same as in Section 4), (ii) with protoplanet radiative feedback only (Run2; same as in Section 4), (iii) with protoplanet and star radiative feedback (tRun $1 ; T(1 \mathrm{au})=250 \mathrm{~K})$, and (iv) only with stellar radiative feedback [tRun 2 with $T(1 \mathrm{au})=250 \mathrm{~K}$; tRun3 with $T(1 \mathrm{au})=150 \mathrm{~K}$ ]. The protoplanet's orbital radius, mass, eccentricity are shown in Fig. B1.

We see by comparing the runs with stellar feedback (tRun 2, tRun3) with the run without any feedback (Run1) that the protoplanet needs more time to open up a gap because the disc is hotter. Therefore, the protoplanet migrates further inward in these runs. When the stellar feedback is not high enough (tRun 3 ), the protoplanet, after opening up the gap, migrates outwards, whereas when the feedback is stronger (tRun 2) the migration continues inwards but at a much longer time-scale. In any case, the mass growth of the protoplanet is slower when the stellar feedback is included (Fig. B1, middle). The eccentricity of the protoplanet is lower (Fig. B1, middle), but if the feedback is not strong enough there is a small eccentricity growth (tRun3).

We also see that there is almost no difference in the evolution of the protoplanet when stellar radiative heating is added on top of the protoplanet radiative heating (compare Run2 and tRun1 in Fig. B1). The effect of the protoplanet feedback is to heat and therefore stabilize the gap edges, so that the feedback from the central star has no additional effect. We conclude that radiative heating from the protoplanet plays a more significant role in the protoplanet's evolution than heating from the central star. 

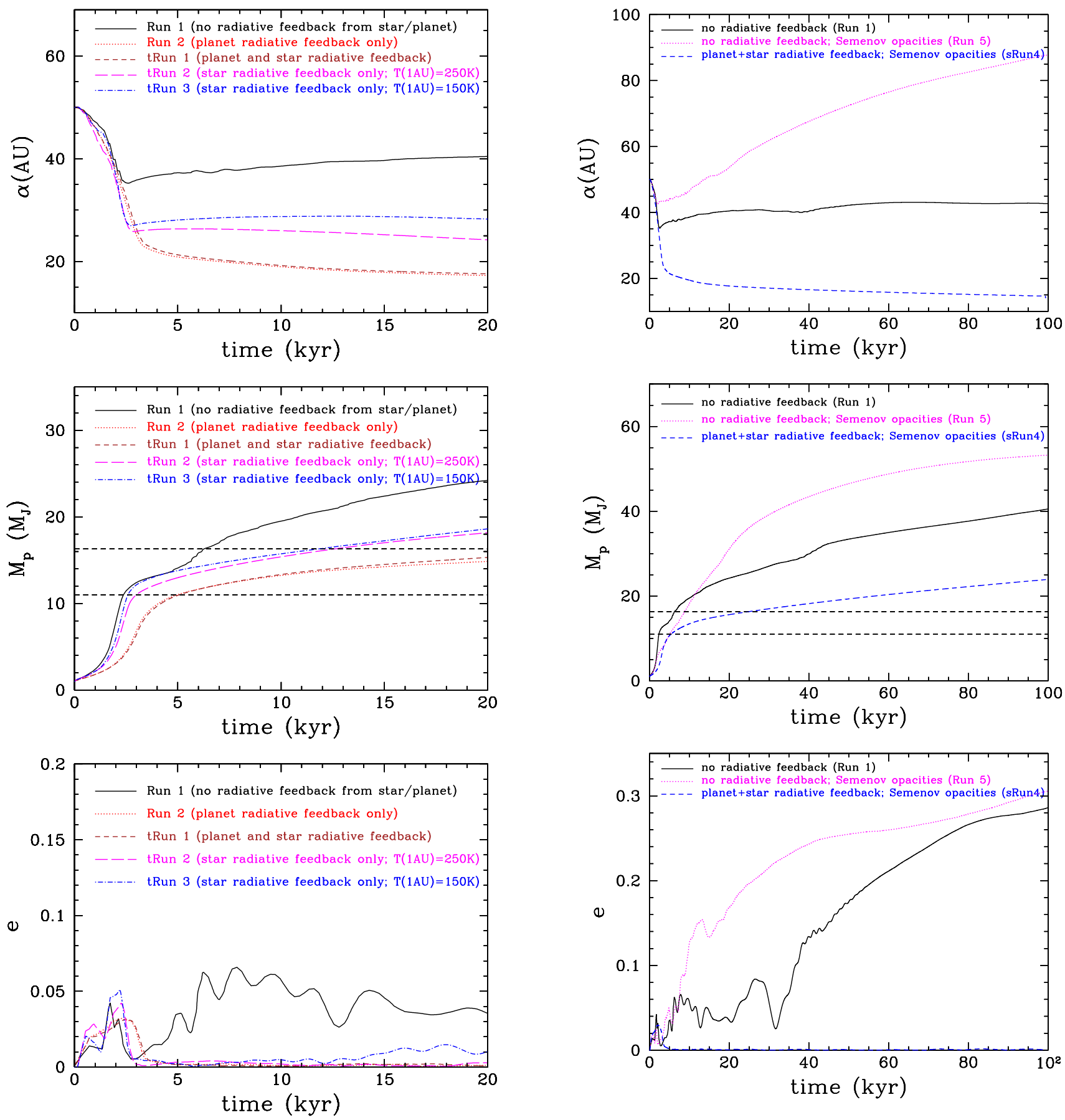

Figure B1. The mass (top), the semimajor axis (middle), and the eccentricity (bottom) of a protoplanet embedded in a disc in five simulations with different types of radiative feedback from the star and the protoplanet, as marked on the graph (see the text for details). Feedback from the protoplanet dominates over the stellar feedback.

\section{APPENDIX C: LONG-TERM PROTOPLANET EVOLUTION}

The simulations presented so far have followed the evolution of the protoplanet in the disc only for $20 \mathrm{kyr}$, i.e. for a relatively short duration compared to the estimated disc lifetimes (a few Myr). In this section, we present simulations in which we follow three representative simulations for much longer, i.e. $100 \mathrm{kyr}$ (see Fig. C1). The details of the simulations are shown in the graphs. These simula-

Figure C1. The long-term evolution of a protoplanet in a disc for four representative simulations. Semimajor axis (top), protoplanet mass (middle), and eccentricity (bottom) are plotted against time.

tions are computationally time-consuming and cannot be performed for all the runs presented in this paper.

In the run with protoplanet radiative feedback (Fig. C1; blue lines), the inward migration continues at a very slow rate and the protoplanet eventually ends up on an orbit with a semimajor axis of about 10 au from the central star. Its mass growth also continues and at the end of the simulation it has become a low-mass brown dwarf (mass $\sim 25 M_{\mathrm{J}}$ ). There is no eccentricity growth in this run and the protoplanets orbit remain circular, as radiative feedback from the protoplanet stabilizes the gap edges. The outcome of this simulation is a low-mass brown dwarf at a close-orbit near its star. 
In the two runs without radiative feedback from the protoplanet (Fig. C1; black and red lines), the protoplanet continues to migrate outwards at a slow pace for the run with the Bell \& Lin (1994) opacities (black line) and at a faster pace for the run with the Semenov et al. (2003) opacities (which are generally larger). In the latter case, the protoplanet has reached a semimajor axis of $\sim 90$ au at the end of the simulation. However, in both runs the protoplanet has grown considerably in mass to become a mid- to high-mass brown dwarf (mass $\sim 40-55 M_{\mathrm{J}}$ ). The objects in the two runs sustain significant eccentricity growth reaching $e \sim 0.3$ at the end of the simulation. The outcomes of these two simulations are high-mass brown dwarfs on wide, eccentric orbits around their stars. Therefore, for a protoplanet to end up as a planet it either needs to accrete at a much lower rate or the disc needs to dissipate within a relatively short time-scale.

This paper has been typeset from a $\mathrm{T}_{\mathrm{E}} \mathrm{X} / \mathrm{AT} \mathrm{T} \mathrm{X}$ file prepared by the author. 